\title{
1 Widening the landscape of transcriptional regulation of algal photoprotection
}

2

\section{Authors}

4 Marius Arend ${ }^{1,2^{\dagger}}$, Yizhong Yuan ${ }^{3 \dagger}$, M. Águila Ruiz-Sola ${ }^{3 \ddagger}$, Nooshin Omranian ${ }^{1,2,4}$, Zoran Nikoloski ${ }^{1,2,4^{*}}$, Dimitris 5 Petroutsos $^{3 *}$

\section{6}

\section{Affiliations}

$8{ }^{1}$ Bioinformatics Group, Institute of Biochemistry and Biology, University of Potsdam, 14476 Potsdam, 9 Germany

*Corresponding Authors: nikoloski@mpimp-golm.mpg.de (Z.N.), dimitris.petroutsos@cea.fr (D.P.)

${ }^{2}$ Systems Biology and Mathematical Modeling Group, Max-Planck-Institute of Molecular Plant Physiology, 14476 Potsdam, Germany

${ }^{3}$ University of Grenoble Alpes, CNRS, CEA, INRAE, IRIG-LPCV, 38000 Grenoble, France

${ }^{4}$ Bioinformatics and Mathematical Modeling Department, Center of Plant Systems Biology and Biotechnology, 4000 Plovdiv, Bulgaria

${ }^{\dagger}$ these authors contributed equally to this work

${ }^{\ddagger}$ Current address: Instituto de Bioquímica Vegetal y Fotosíntesis, Universidad de Sevilla-CSIC, 41092, Sevilla, SPAIN 


\section{Abstract}

24 Availability of light and $\mathrm{CO}_{2}$, substrates of microalgae photosynthesis, is frequently far from optimal. 25 Microalgae activate photoprotection under strong light, to prevent oxidative damage, and the $\mathrm{CO}_{2}$ 26 Concentrating Mechanism (CCM) under low $\mathrm{CO}_{2}$, to raise intracellular $\mathrm{CO}_{2}$ levels. The two processes are 27 interconnected; yet, the underlying transcriptional regulators remain largely unknown. By employing a large 28 transcriptomics data compendium of Chlamydomonas reinhardtii's responses to different light and carbon 29 supply we reconstructed a consensus genome-scale gene regulatory network from complementary inference 30 approaches and used it to elucidate the transcriptional regulation of photoprotection. We showed that the

31 CCM regulator LCR1 also controls photoprotection, and that QER7, a Squamosa Binding Protein, suppresses 32 photoprotection- and CCM-gene expression under the control of the blue light photoreceptor Phototropin.

33 Along with demonstrating the existence of regulatory hubs that channel light- and $\mathrm{CO}_{2}$-mediated signals into 34 a common response, our study provides a unique resource to dissect gene expression regulation in this 35 microalga. 


\section{Introduction}

Photosynthetic microalgae convert light into chemical energy in the form of ATP and NADPH to fuel the $\mathrm{CO}_{2}$ fixation in the Calvin-Benson cycle ${ }^{1}$. They have evolved to cope with rapid fluctuations in light ${ }^{2}$ and inorganic carbon ${ }^{3}$ availability in their native habitats. When absorbed light exceeds the $\mathrm{CO}_{2}$ assimilation capacity, the formation of harmful reactive oxygen species can lead to severe cell damage; this is prevented by the activation of photoprotective mechanisms, collectively called non-photochemical quenching (NPQ). NPQ encompasses several processes that are distinguished in terms of their timescales ${ }^{2}$, among which the rapidly reversible energy-quenching ( $\mathrm{qE}$ ) is, under most circumstances, the predominant NPQ component ${ }^{2,4}$. The major molecular effector of $q E$ in Chlamydomonas is the LIGHT HARVESTING COMPLEX STRESS RELATED protein LHCSR3, encoded by the LHCSR3.1 and LHCSR3.2 genes ${ }^{5}$ that slightly differ only in their promoters; LHCSR1 can also contribute significantly in qE under conditions where LHCSR3 is not expressed ${ }^{6,7}$. PSBS, the key qE effector protein in higher plants ${ }^{8}$ is encoded as two highly similar paralogues PSBS1 and PSBS2 in Chlamydomonas ${ }^{9}$. They are only transiently expressed in Chlamydomonas under high light (HL) $)^{9,10}$ and accumulate under UV-B irradiation ${ }^{11}$; their precise contribution in Chlamydomonas photoprotective responses is still unresolved ${ }^{12}$.

Intracellular levels of $\mathrm{CO}_{2}$ are modulated by the availability of its gaseous and hydrated forms ${ }^{3}$ in the culture media and the supply of acetate, that is partly metabolized into $\mathrm{CO}_{2}{ }^{7,13}$. Under low $\mathrm{CO}_{2}$, Chlamydomonas activates the $\mathrm{CO}_{2}$-concentrating mechanism (CCM) to avoid substrate-limitation of photosynthesis by raising the $\mathrm{CO}_{2}$ concentration at the site of RuBisCO, where $\mathrm{CO}_{2}$ is assimilated ${ }^{3}$. The $\mathrm{CCM}$ mainly comprises of carbonic anhydrases (CAHs) and of inorganic carbon transporters. Almost all CCM-related genes are under the control of the nucleus-localized zinc-finger type nuclear factor CIA5 (aka CCM1) ${ }^{14-16}$, including the Myb Transcription Factor LOW-CO - -STRESS RESPONSE 1 (LCR1) that controls expression of genes coding for the periplasmic CAH1, the plasma membrane-localized bicarbonate transporter LOW CO${ }_{2}$ INDUCED 1 (LCI1), and the low- $\mathrm{CO}_{2}$ responsive $\mathrm{LCl} 6$, whose role remains to be elucidated ${ }^{17}$. $\mathrm{ClA} 5$ is also a major qE regulator activating transcription of genes encoding LHCSR3 and PSBS, while repressing accumulation of LHCSR1 protein $^{7}$.

LHCSR3 expression relies on blue light perception by the photoreceptor phototropin (PHOT) ${ }^{18}$, on calcium signaling, mediated by the calcium sensor $\mathrm{CAS}^{19}$ and on active photosynthetic electron flow ${ }^{18-20}$, likely via indirectly impacting $\mathrm{CO}_{2}$ availability ${ }^{7}$. The critical importance of $\mathrm{CO}_{2}$ in LHCSR3 expression is demonstrated by the fact that changes in $\mathrm{CO}_{2}$ concentration can trigger LHCSR3 expression ${ }^{21-23}$ even in the absence of light ${ }^{7}$. Accumulation of LHCSR1 and PSBS mRNA is under control of the UV-B photoreceptor UVR8 ${ }^{11}$ and $\mathrm{PHOT}^{24,25}$ 
and is photosynthesis-independent ${ }^{20,25}$. While LHCSR1 is $\mathrm{CO}_{2} / \mathrm{CIA} 5$ independent at the transcript level ${ }^{7,25}$, PSBS is responsive to $\mathrm{CO}_{2}$ abundance and is under partial control of $\mathrm{CIA5}^{7}$. A Cullin (CUL4) dependent E3-ligase $24,26,27$ has been demonstrated to post-translationally regulate the transcription factor (TF) complex of CONSTANS $(\mathrm{CrCO})^{26}$ and NF-Y isomers ${ }^{27}$, which bind to DNA to regulate the transcription of LHCSR1, LHCSR3, and PSBS. The putative TF and diurnal timekeeper RHYTHM OF CHLOROPLAST 75 (ROC75) was shown to repress LHCSR3 under illumination with red light ${ }^{28}$.

Here, we employed a large compendium of RNAseq data from Chlamydomonas to build a gene regulatory network (GRN) underlying light and carbon responses, and thus reveal the transcriptional regulation of $q E$ at the interface of these responses. The successful usage of RNAseq data to infer GRNs has been demonstrated in many studies ${ }^{29-31}$, although the data pose some challenges that require careful consideration. All of the developed approaches to infer a GRN quantify the interdependence between the transcript levels of TFcoding genes and their putative targets; the resulting prediction model serves as a proxy for the regulatory strength that the product of the TF-coding gene exerts on its target(s). It is usually the case that the number of observations (samples) is considerably smaller than the number of TFs used as predictors, leading to collinearity of the transcript levels and associated computational instabilities; further, as an artifact of the computational techniques, some of the inferred regulations may be spurious ${ }^{32-34}$. To address these issues, here we took advantage of combining the outcome of multiple regularization techniques and post-processing to increase the robustness of identified interactions ${ }^{33-35}$. In contrast to our approach, the existing predicted GRNs of Chlamydomonas either focused on nitrogen starvation ${ }^{36}$ or used a broad RNAseq data compendium, not tailored to inferring regulatory interactions underlying responses to particular cues ${ }^{37}$. Moreover, these GRNs were not obtained by combining the outcomes from multiple inference approaches, shown to increase accuracy of predictions ${ }^{30}$, and their quality was not gauged against existing knowledge of gene regulatory interactions.

We used an RNAseq data compendium of 158 samples (Supplementary Table 1) from Chlamydomonas cultures exposed to different light and carbon supply as input to seven benchmarked GRN inference approaches that employ complementary inference strategies ${ }^{29,30}$. We assessed the performance of each approach based on a set of curated TF-target gene interactions with experimental evidence from Chlamydomonas. Based on this assessment, we integrated the outcome of the five best performing approaches into a unique resource, a consensus network of Chlamydomonas light- and carbon-dependent transcriptional regulation. We used the consensus network to reveal regulators of $\mathrm{qE}$ genes and demonstrated the quality of predictions by validating two of the six tested candidates. We showed that LCR1 
acts as a qE regulator by activating the expression of LHCSR3, and demonstrated that qE-REGULATOR 7 (QER7) is a repressor of $\mathrm{qE}$ and $\mathrm{CCM}$-gene expression. Our work consolidates the extensive co-regulation of $\mathrm{CCM}$ and photoprotection ${ }^{7}$ based on the untargeted assessment of the obtained genome-scale GRN.

\section{Results}

\section{Computationally inferred GRN recovered known regulatory interactions underlying qE and CCM in} Chlamydomonas

We first aimed to employ published and here generated RNAseq data sets capturing the transcriptional responses of Chlamydomonas to light and acetate availability to infer the underlying GRN. To this end, we obtained data from two publicly available transcriptomics studies of synchronized chemostat wild-type (WT) cultures grown in a $12 \mathrm{~h} / 12 \mathrm{~h}$ light dark scheme and sampled in $30 \mathrm{~min}$ to $2 \mathrm{~h}$ intervals $\mathrm{s}^{38,39}$. We combined these with our RNAseq data generated from mixotrophically or autotrophically grown batch cultures of the WT and phot mutant acclimated to low light (LL) or exposed to HL (Methods, Supplementary Table 1). These data sets capture the expected expression patterns of the key genes involved on CCM and qE (Fig. 1a) in response to changes in acetate availability and light intensity. Specifically, we found strong up-regulation of these genes in the light ${ }^{7,25}$, and a marked inhibition of LHCSR3.1/2 and CCM genes by acetate as previously described ${ }^{7,40}$.

We employed these data together with a list of 407 transcription factors from protein homology studies ${ }^{41,42}$ (Methods, Supplementary Table 2), as input to seven GRN inference approaches to robustly predict TF-target interactions, as shown in benchmark studies ${ }^{30}$. Since there exists no study that experimentally probes TF binding to DNA on a genome-scale, we first curated a list of known, experimentally validated regulatory interactions underlying CCM and qE in Chlamydomonas ${ }^{22,26-28}$, to assess the quality of GRNs inferred by the different approaches (Fig. 1b). As a negative control, we considered the lack of effect of the SINGLET OXYGEN RESISTANT 1 (SOR1) TF on PSBS1 transcript levels in diurnal culture ${ }^{43}$. The comparative analysis of the predicted and known interactions demonstrated that two of the applied approaches for GRN inference (i.e. $\mathrm{ARACNE}^{44}$ and global silencing ${ }^{45}$ ) are unable to recover any literature interactions when using a network density threshold of $10 \%$ of all possible TF-TF and TF-target interactions. This poor performance is due either to over-trimming or issues with the validity of the underlying assumptions, as seen in other case studies ${ }^{46}$. We therefore considered only the remaining five approaches, namely: Graphical Gaussian Models (GGM), Context Likelihood of Relatedness (CLR), Elastic Net regression, Gene Network Inference with Ensemble of Trees (GENIE3), and Network Deconvolution to infer a consensus GRN by using the Borda count election method $^{30,47}$ (Methods). We then ranked the inferred interactions within each approach and quantified the 
variability of ranks for the known TF-target gene interactions. We found that the average standard deviation of the ranks of the TF-target gene interactions within an approach is larger than the average standard deviation for the rank of a TF-target gene interaction across the five approaches (Extended Data Fig. 1, Fig. 1b). This observation suggested that the properties of a given TF-target gene interaction have a stronger influence on its assigned rank than the inference approach used. More specifically, we noted that the regulation of LHCSR genes by the two NF-Y paralogues and the induction of LCR1 by CIA5 are not recovered by any of the used approaches; this is in line with reports showing that CIA5 is constitutively expressed and regulated post-translationally ${ }^{16}$-not reflected in the transcriptomics data. Further, NF-Y factors that rely on complex formation with $\mathrm{CrCO}$ to regulate their targets ${ }^{27}$, may also act via unresolved posttranslational mechanisms. Importantly, the regulatory interactions of the CCM effector genes $L C I 1$ and CAH1 by LCR 1 are assigned very high ranks (top 1\%) by the employed approaches (except for GGM) considered in the consensus GRN; moreover, the experimentally falsified interaction of SOR1 and PSBS1 transcripts ${ }^{43}$ is correctly discarded by all approaches (Fig. 1b). In addition, we observed that CLR and GENIE3 demonstrated the best performance with respect to the set of known interactions. For instance, they identified the regulation of LHCSR3.1 by $\mathrm{CrCO}^{26,27}$ and of PSBS1 by NF-YB ${ }^{27}$ (Fig. 1b). Generalization of this ranking beyond the known interactions underlying $\mathrm{qE}$ and $\mathrm{CCM}$ processes is challenging, due to the lack of genome-scale gold standard, and we therefore opted to combine the results of the five approaches, that showed comparable performance, in the of the overlap between the consensus and individual GRNs and the enrichment of TF-TF interactions demonstrated the robustness of the inferred interactions (Supplementary Note 1, Extended Data Fig. 2).

\section{Consensus GRN pinpoints LCR1 as a regulator of qE-related genes}

150 Using the consensus GRN, we inferred direct regulators of LHCSR and PSBS genes ) and ranked them according to the score resulting from the Borda method (Methods) ${ }^{30,47}$. Mutants were available for four of the top ten knock-out mutants of previously uncharacterized genes were ordered from the Clip library ${ }^{48}$, which we termed qE-regulators 4 and 6 (qer4, qer6; see Extended Data Fig. 3 for the genotyping of these mutants). Additionally, we obtained an over-expressor line of the $\mathrm{N}$-acetyltransferase $\mathrm{LCl}^{49}$ and the knock-out strain of the known CCM regulator LCR1 ${ }^{17}$. We tested for a regulatory effect by switching LL-acclimated mutant strains and their respective WT background to HL for $1 \mathrm{~h}$ and quantified transcript levels of qE-related genes. For qer4, qer6, and Ici8-oe we could not observe an effect on the transcript levels of investigated genes after $\mathrm{HL}$ 
despite the fact that qer4 accumulated 1.5 times more LHCSR3.1 under LL than the WT. A review of the closest

161

162

163

164

165

166

167

168

169

170

171

172

173

174

175

176

177

178

179

180

181

182

183

184

185

186

187

188

189

190

orthologs of $\mathrm{LCl} 8$ together with the experimental data indicate that it is likely involved in arginine synthesis ${ }^{49}$ and wrongly included as histone acetylase in the list of TFs. Interestingly, LCR1, the highest ranking among the tested regulators showed significantly decreased expression of LHCSR3 at both the gene (3 times lower, Fig. 2b) and protein level (4 times lower, Fig. 2c,d) compared to the WT; as a result, Icr1 developed very low NPQ and qE (Fig. 2e). Complementation of Icr1 with the knocked-out gene (strain Icr1-C) restored LHCSR3 gene and protein expression as well as the qE phenotype (Fig. 2b-e). Interestingly, the Icr1 mutant overaccumulated LHCSR1 and PSBS both at the transcript and the protein level (Fig. 2b-d); Complementation of Icr1 with the knocked-out gene (strain Icr1-C) restored LHCSR3 gene and protein expression as well as the qE phenotype (Fig. 2b-e). Because pre-acclimation conditions impact qE gene expression ${ }^{25}$ we conducted independent experiments in which cells were acclimated to darkness before exposure to HL and we obtained very similar results. Our data demonstrated that Icr1 showed significantly lower expression of LHCSR3 and higher expression of LHCSR1/PSBS at both the gene (Extended Data Fig. 5a) and protein level (Extended Data Fig. 5b,c), and had lower NPQ and qE (Extended Data Fig. 5d) than the WT, although the higher expression levels of LHCSR1 gene were not rescued by the complementation with the missing LCR1 gene (Extended Data Fig. 5a). Altogether, our data show that LCR1 is a regulator of qE by activating LHCSR3.1 transcription and repressing LHCSR1 and PSBS accumulation.

Further, we revisited the role of LCR1 in regulating CCM genes ${ }^{17}$ by analyzing expression of selected CCM genes in WT, Icr1 and Icr1-C cells shifted from LL or darkness to HL, conditions favoring CCM gene expression?. We first confirmed that under our experimental conditions Icr1 could not fully induce LCI1 (Extended Data Fig. $6 a, b)$ in accordance to the report of the discovery of LCR1 ${ }^{17}$. Our analyses further showed a statistically significant impairment of Icr1 in inducing genes encoding the Ci transporters LOW-CO - INDUCIBLE PROTEIN A (LCIA), HIGH-LIGHT ACTIVATED 3 (HLA3), CHLOROPLAST CARRIER PROTEIN 1 (CCP1) and BESTROPHINE-LIKE PROTEIN 1 (BST1) as well as the carbonic anhydrase CAH4, when shifted from LL or dark to HL (Extended Data

Fig. $6 \mathrm{a}, \mathrm{b})$, indicating that the role of LCR1 in low- $\mathrm{CO}_{2}$ gene expression extends beyond the regulation of gene expression of $C A H 1, L C I 1$ and $L C I 6^{17}$.

\section{PHOT-specific GRN reveals a novel repressor of qE and CCM}

The light-dependent induction of LHCSR3 is predominantly mediated by the blue light photoreceptor PHOT $^{18}$. To analyze the PHOT-dependent transcriptional regulators, we first identified the interactions shared by the consensus network and a GRN inferred only by GENIE3, due to its good performance, using the RNAseq data from samples of phot and WT acclimated to LL and after $1 \mathrm{~h}$ exposure to HL (Methods). This so-called PHOT- 
specific GRN (Methods, Supplementary Table 5) comprises regulatory interactions underlying the transcriptomic changes observed in the phot mutant while burrowing the statistical power of the RNAseq compendium.

When investigating the top 10 regulators of $\mathrm{qE}$ genes in this PHOT GRN the interactions where weighted based on the importance score from GENIE3 (Supplementary Table 6, Fig. 3a). Among these interactions we recovered the previously discussed false positive LCl8 gene (see above, Fig. 3a) (Extended Data Fig. 4). In addition, two previously reported regulators of $\mathrm{qE}, \mathrm{ROC}^{28}$ and $\mathrm{CrCO}^{26,27}$, are also included in this list of PHOT-dependent regulators. These observations are in line with an existing hypothesis ${ }^{24}$ suggesting that a CUL4-dependent E3-ligase targeting $\mathrm{CrCO}^{, 26}$ acts downstream of PHOT. ROC75 has been previously reported to act independently of the PHOT signal based on qPCR studies of the mutant grown synchronously under different light spectra ${ }^{28}$. In our RNAseq data, gathered under continuous white light, we observed a significant difference in expression levels of ROC75 between WT and phot $(\log 2$ fold-change $=1.03$, adj. $p$-value $=$ $\left.1.80^{*} 10^{-7}\right)$.

The fact that several regulators showed larger regulatory strength than $\mathrm{CrCO}$ in the PHOT GRN indicates the existence of yet unreported regulators of qE effector genes in the PHOT signaling pathway. This is in line with existing results ${ }^{26}$, showing that the knock-out of $\mathrm{CrCO}$ is insufficient to fully abolish light-dependent activation of LHCSR3. Following this reasoning we obtained ger1 and qer7, the available regulator candidates mutants, from the CLiP library ${ }^{48}$, (for genotyping see Extended Data Fig. 3). Our results show higher mRNA and protein levels of LHCSR1 in the qer1 mutant (Extended Data Fig. 7); however, this could not be rescued by ectopic expression of the QER1 gene in the qer1 mutant background (Extended Data Fig. 7). We found significant upregulation of LHCSR3.1 gene expression in the qer7 mutant (1.7 times, Extended Data Fig. 8a) also reflected in higher NPQ (Extended Data Fig. 8b) and qE levels (Extended Data Fig. 8c) which we followed up in more detail. To this end, we ectopically expressed the WT QER7 gene in the ger7 mutant and generated the complemented strain qer7-C that expressed QER7 to levels similar to those WT (Extended Data Fig. 3c). As a result, the ger7-C strain showed reduced LHCSR3 gene expression, NPQ and qE levels as compared with the qer7 mutant (Extended Data Fig. 8a-c). LHCSR1 and PSBS seemed to be unaffected in the qer7 in these LL to HL transition experiments (Extended Data Fig. 8a). As with LCR1, we also performed dark to HL experiments to further characterize the photoprotective responses of qer7; under these conditions, qer7 accumulated significantly more LHCSR1 (1.7 times) and PSBS1 (2.2 times) while LHCSR3 remained unaffected (Extended 
(LHCSR1, PSBS, NPQ, qE; Extended Data Fig. 8d-f). These data validate the prediction of QER7 as regulator of qE gene expression (Fig. 3a) and indicate that QER7 regulates different subsets of qE genes depending on the pre-acclimation conditions; LHCSR3 when preacclimation is under LL, LHCSR1 and PSBS when pre-acclimation occurs in darkness. Motivated by these findings and given the fact that most of the Chlamydomonas transcriptome, undergoes diurnal changes according to biological function ${ }^{39}$ we decided to address the role of QER7 in regulating qE genes under light/dark cycles. We synchronized WT, qer7 and qer7-C cells in $12 \mathrm{~h}$ $\mathrm{L} / 12 \mathrm{~h} \mathrm{D}$ cycle and exposed them to $\mathrm{HL}$ right after the end of the dark phase. Our results revealed that under these conditions QER7 functions as a repressor of all qE-related genes; the qer7 mutant expresses significantly higher LHCSR3, LHCSR1 and PSBS both at the gene (Fig. 3b) and protein (Fig. 3c,d) level, and exhibits higher NPQ and qE (Fig. 3e), with all phenotypes rescued in the qer7-C complemented line. Previous protein homology studies identified QER7 as Squamosa Binding Protein ${ }^{50}$ or bZIP TF ${ }^{51}$, and here, we provide the first functional annotation of QER7 as a novel qE regulator.

\section{QER7 co-regulates qE-related and CCM genes}

235

236

237

238

239

240

241

242

The recent findings that the regulatory role of $\mathrm{CIA}^{7}$ and LCR1 (Fig. 2 and Extended Data Fig. 5) extends beyond CCM to also control qE-related gene expression, prompted us to also inspect the expression levels of CCM genes in synchronized qer7 cells (Fig. 4a). Indeed, for four of these transcripts (CAH4, LCIA, CCP1, HLA3) we observed a significant upregulation in qer7 after $\mathrm{HL}$ exposure that was reversed by complementation with the QER7 gene, indicating that QER7 supresses expression of CCM genes; the suppression role of QER7 on CCM genes was only observable under $\mathrm{HL}$, conditions that favor CCM gene expression ${ }^{7}$ and not under LL (Fig. 4a). Interestingly, and in line with QER7 acting as suppressor of CCM gene expression (Fig. 4a), we found LCR1 and QER7 among the top 10 regulators of the CCM genes probed by qPCR in the inferred GRNs (Extended Data Fig. 9).

After observing the inhibition of CCM gene transcription by QER7, we investigated the signaling pathway upstream of QER7. To this end, we quantified QER7 gene expression in synchronized phot cultures. As expected, we observe a strong effect of the PHOT knock-out showing that PHOT suppresses QER7 expression (Fig. 4b) implying that blue-light perception via PHOT is involved in the regulation of CCM related gene expression. Interestingly, phot expressed LCR1 to levels similar to those in WT (Fig. 4c). Thus, while sharing part of their target genes, the two TFs mediate different signals. These data demonstrate that the PHOTspecific GRN successfully pinpoints TFs that depend on PHOT activity while retaining the statistical power to recover genuine regulatory interactions. 
The two $\mathrm{qE}$ regulators that we validated in this study also regulate CCM genes. Therefore, we next investigated to what extent the observed coregulation pattern applies to the global, known transcriptional regulation of low $\mathrm{CO}_{2}$ and light stress responsive genes. To this end, we took advantage of the size of the presented genome-scale GRNs and compiled a list of genes putatively involved in photoprotection strongest regulatory strength on the genes in the compiled lists. We found six (empirical p-value $<0.001$, Methods) and four (empirical p-value $<0.01$ ) of the top 10 regulators to be shared between these two responses in the consensus (Fig. 5a,b) and the PHOT-specific GRN, respectively (Figure 5c,d). The significant, assimilatory processes is a principal feature of Chlamydomonas' transcriptional regulatory program.

\section{Discussion}

263

264

265

266

267

268

269

270

271

272

273

274

275

276

277

278

279

280

The molecular actors and structure of the transcriptional regulatory mechanisms that shape Chlamydomonas' response to differential light and carbon availability are largely unknown, although they are paramount to survival of Chlamydomonas and offer valuable targets for biological engineering. Here we set out to elucidate the GRN underlying the response to light and carbon availability by combining the results from five complementary inference approaches and data from 158 RNAseq samples of cultures responding to these cues. The obtained GRN enabled us to identify novel regulators controlling qE. Experimentally testing available mutant strains for six of these candidates we were able to validate two TFs: QER7, suppressing qEand CCM-gene expression, and LCR1, activating LHCSR3 and suppressing LHCSR1 and PSBS expression. The two TFs belong to independent signalling pathways: LCR1 has been known to controlled by CIA5 while we found that QER7 is controlled by Phototropin; this last finding adds a new layer of regulation of CCM by blue light sensing. We complemented the finding of the involvement of LCR1 in the regulation of qE related genes with an analysis of the genome-scale co-regulation of CCM and photoprotective genes. Indeed, we observed significant number (six out of the top 10) of regulators targeting both processes in the inferred consensus GRN. Together with previous studies, our results provide evidence for extensive signaling crosstalk between light and carbon sensing and name a set of TFs that likely integrate these disparate signals into a common transcriptional response. The GRN gives an unbiased representation of the genome-scale regulators acting in light and carbon sensing. Thus, it represents a powerful resource for future dissection of the transcriptional regulation of responses of Chlamydomonas to light and carbon availability. 


\section{Methods}

283

284

285

286

287

288

289

290

291

292

293

294

295

296

297

298

299

300

301

302

303

304

305

306

307

308

309

310

311

\section{Transcriptome analysis}

We assembled a compendium of RNAseq data (Supplementary Table 1) that capture regulation of lightdependent processes by combining in-house produced RNAseq measurements with publicly available data from two studies of densely sampled diurnal cultures of Chlamydomonas ${ }^{38,39}$. For the samples in the acetate time-resolved experiment, adapter sequences were specifically trimmed from raw reads using BBduk 52 ( $k$ trim=r k=30 mink=12 minlen=50). Raw reads of the diurnal transcriptome study from Strenkert et al. ${ }^{39}$ were obtained from NCBI GEO database (GSE112394). Reads were aligned to the Chlamydomonas reference transcriptome ${ }^{53}$ available from JGI Phytozome (Assembly version 5) using RNA STAR aligner. The BAM files obtained from these measurements were analyzed using HTSeq-count ${ }^{54}$ (standed=reverse) to create raw read count files. The raw read counts from Zones et al. ${ }^{38}$ were obtained as .tsv from NCBI GEO (GSE71469). The final data set consists of 158 samples from 62 experimental conditions or time points (Supplementary Table 1). Genes with less than 1 count per million in at least 9 measurements where discarded and the remainder were voom ${ }^{53}$ transformed and normalized using library normalization factors based on the $\mathrm{TMM}^{55}$ approach as implemented in the R Bioconductor package edge $\mathrm{R}^{56}$.

\section{Transcription factor set from comparative genomics}

To reduce the set of parameters in our network model, we compiled transcription factor (TF) annotations for the Chlamydomonas genome based on proteome homology studies. We obtained the proteomes and protein IDs of predicted Chlamydomonas TFs from Pérez-Rodríguez et al. ${ }^{41}$ Since these predictions were built based on the older Chlamydomonas assembly, we first used the conversion table provided by Phytozome to convert JGI4 to Crev5.6 IDs. For the TFs that could not be recovered by this approach we used the Phytozome BLAST tool to align these sequences against the Crev5.6 proteome (BLASTP, E threshold: -1, comparison matrix: BLOSUM62, word length: 11, number of reported alignments: 5). The reported hits were filtered for sequence identity $>97 \%$ and gaps $\leq 1$. If sequences mapped multiple times to the same Crev5.6 gene ID, only the hit alignment closest to the $\mathrm{N}$-terminus of the query sequence was kept. The hit was only accepted if the alignment started at least six residues from the $\mathrm{N}$-terminus of the hit sequence. For Crev5.6 loci that had multiple JGI4 TF queries assigned to them the best hit was selected manually. This set was then extended by the TFs found in the study of Jin et al. ${ }^{42}$ and the regulators in the manually curated set of CCM and $\mathrm{qE}$ regulatory interactions (Supplementary Tables 7 and 8). Using this procedure, we compiled a list of 407 Chlamydomonas TFs (Supplementary Table 2) to be considered as regulators in the inferred networks. 


\section{Gene regulatory network inference}

313 The CLR and ARACNE approach were based on all replicate measurements; for all other inference methods 314 the median from each condition was used as input. All input matrices where standardized gene-wise. If not explicitly stated in the respective paragraph the implementations of all GRN inference approaches were applied with their default settings.

\section{Graphical Gaussian Models}

318 The network inferred from a Graphical Gaussian model of gene regulation was obtained using the implementation of the partial correlation estimate from Schäfer et al. ${ }^{34}$ as implemented in the R GeneNet package. All interactions between TFs and another gene/TF with non-zero partial correlations were included as network edges.

\section{GENIE3}

The random forest-based network from GENIE3 was generated using the R Bioconductor implementation provided by the authors ${ }^{57}$. We used only expression levels of TFs as predictors.

\section{Elastic net regression}

A linear regression based network was obtained using the elastic net algorithm ${ }^{35}$. A model was fit for each gene using the expression levels of all TFs as predictors. The two hyperparameters $\lambda 2$ (quadratic penalty) and s (fraction of L1 norm coefficients) were tuned for each gene model using 6-fold cross validation. The 2D $s=\{0.1,0.2,0.3,0.4,0.5,0.6,0.7,0.8,0.9\}$. The $R 2$ value for each model was calculated as

$$
R^{2}=1-\frac{\sum(y-\hat{y})}{\operatorname{var}(y)}
$$

with $y$ marking the vector of observed expression values and $\hat{y}$ the model predictions. Models with a negative $R 2$ value were discarded as regularization artifacts. The results of the remaining models were assembled into absolute coefficient.

$$
\beta_{n}=\frac{\beta}{\max |\beta|}
$$


337 The implementation of mutual information (MI)-based network inference approaches from the R package 338 minet $^{58}$ was used. Pairwise MI was estimated based on the Spearman correlation as proposed by Olsen et 339 al. ${ }^{59}$. Two networks were constructed based on these MI estimates. Using the CLR approach ${ }^{60}$ non-significant 340 interactions were removed based on the z-scores calculated from the marginal distributions of MI values for 341 each gene pair. Alternatively, the ARACNE algorithm ${ }^{44}$ was used to prune the network based on the data 342 processing inequality. For both networks only interactions originating from a TF were taken into consideration and edges were ranked according to the assigned MI value.

\section{Deconvolution and Silencing}

345 For the two networks based on decomposition of the interaction matrix $G$ the Pearson correlation matrix obtained from gene expression values was used as input.

347 The deconvolution approach introduced by Feizi et al. ${ }^{61}$ was implemented as previously described ${ }^{46}$. The eigenvalue scaling factor $\beta$ was initialized as $\beta=0.9$ and iteratively reduced in increments of 0.05 until the largest eigenvector of the direct interaction matrix generated by deconvolution was smaller than 1. Edges were ranked according to the deconvoluted interaction matrix.

351 The Silencing approach as described by Barzel et al. ${ }^{45}$ was implemented in R. The proposed approximation of the direct interaction matrix $S$ in which spurious interactions are silenced relies on the invers of the observed correlation matrix $G$. In our implementation we used the Moore-Penrose pseudoinverse in case $G$ was close to singular. In the resulting network edges were ranked according to the approximated silenced interaction matrix.

\section{Consensus network construction}

357 To improve network quality ${ }^{30}$, we built a consensus network integrating the GRN models inferred by the different approaches introduced above. To this end, we used the Borda count election method ${ }^{47}$ whereby the rank $r$ of an interaction $I$ in the consensus network built on the predictions from $k$ approaches is given by arithmetic mean of the ranks in the individual networks

$$
r_{\text {consens }}(I)=\frac{\sum_{i=1}^{k} r_{i}(I)}{k}
$$

361 Following the reasoning of Feizi et al. ${ }^{61}$ ) in this integration only the top $10 \%$ all possible edges in the GRN (625815) were considered from each individual ranking. For an edge that was not assigned a rank by some approaches, the missing ranks were set to $10 \%$ of all possible edges plus one. 
Using this integration method, we assembled a consensus network based on all approaches to compare predictions from all GRN inference approaches (Extended Data Fig. 1). Due to this comparison and inability to recover known interactions (Fig. 1b) the rankings derived from ARACNE and Silencing were only considered in Extended Data Fig. 1 and excluded from the final consensus network used for all other analyses. As with the individual networks returned by the different approaches the consensus network (Supplemental Table 3) was trimmed to the top $10 \%$ of all possible edges according to the integrated ranks. The weight of edges in the final network was set as $r_{\text {consens. }}^{-1}$.

\section{PHOT-specific network}

372 To investigate the PHOT-specific regulatory interactions genes that are differentially expressed between phot mutant and wt under low and high light were inferred. To this end, transcript counts of genes with more than 1 count per million in at least four replicates from these conditions (Supplementary Table 1) were tested for differential expression using the R packages limma ${ }^{62}$, DeSeq $2^{63}$, and edge ${ }^{56}$. Only genes deemed significant by all three tools after Benjamini-Hochberg correction for a false discovery rate of 0.05 were considered differentially expressed with respect to PHOT mutation

In the next step, we focused on the normalized and scaled expression levels from these differentially expressed genes and the previously mentioned conditions, to infer a PHOT-specific GRN using GENIE3. To improve robustness of this network, which was obtained from a comparably sparse data set, we only considered the edges in the intersection with the final consensus network. Again, for both networks only the top $10 \%$ of possible edges were taken into account. Therefore, the obtained PHOT-network represents a subnetwork of the final consensus in which edges are weighted by the "PHOT specific" GENIE3 importance measure (Supplementary Table 5).

\section{Identification of major regulators}

We compiled a manually curated list of possible target genes known to be involved in the processes of $\mathrm{qE}$ the regulatory strength of a candidate regulator (for the given process) was determined by the sum of edge weights $w_{i j}$ between this regulator and the $k$ genes in the respective target gene set

$$
C(T F)=\sum_{j=1}^{k} w_{T F j} .
$$


The one-sided $p$-value for the overlap between the regulators of CCM and photoprotective genes was approximated by sampling the overlaps of random gene sets. To this end, we compiled two gene sets with the same cardinality as the curated CCM and photoprotective genes. The genes in these sets where randomly sampled without replacement from all targets in the respective networks. The 10 strongest regulators of these two gene sets where then obtained as previously described and the overlap was calculated as our sample statistic. This process was repeated 10,000 times and an empirical p-value was calculated from the number of iterations, $r$, where the overlap was higher or equal to the observed value, and the total number of iterations, $n^{64}$ :

$$
p=\frac{r+1}{n+1}
$$

\section{Strains and conditions}

401

402

403

404

405

406

407

408

409

410

411

412

413

414

415

416

417

C. reinhardtii strains were grown under $15 \mu \mathrm{mol}$ photons $\mathrm{m}^{-2} \mathrm{~s}^{-1}$ ) in Tris-acetate-phosphate (TAP) media ${ }^{65}$ at $23^{\circ} \mathrm{C}$ in Erlenmeyer flasks shaken at $125 \mathrm{rpm}$. For all experiments cells were transferred to Sueoka's high salt medium (HSM) ${ }^{66}$ at 1 million cells $\mathrm{mL}^{-1}$ and exposed to light intensities as described in the text and figure legends. For the investigation of the impact of acetate on the genome-wide transcriptome, HSM was supplemented with $20 \mathrm{mM}$ sodium acetate. C. reinhardtii strain CC-125 mt+ was used as WT. The phot (depleted from PHOT1; gene ID: Cre03.g199000), was previously generated ${ }^{67}$ and recently characterized ${ }^{25}$. For synchronized cultures, the cells were grown in HSM for at least 5 days under a $12 \mathrm{~h}$ light/12h dark cycle (light intensity was set at $15 \mu \mathrm{mol}$ photons $\mathrm{m}^{-2} \mathrm{~s}^{-1}$; temperature was $18{ }^{\circ} \mathrm{C}$ in the dark and $23{ }^{\circ} \mathrm{C}$ in the light). All CLiP mutant strains used in this study and their parental strain (CC-4533) were obtained from the CLiP library (REF); qer1 (LMJ.RY0402.072278), qer4 (LMJ.RY0402.202963), qer6 (LMJ.RY0402.162350), qer7 (LMJ.RY0402.118995). The Icr1 (strain C44), Icr1-C (strain C44-B7) and its parental strain Q30P3 as described in ${ }^{17}$ were a kind gift from Hideya Fukuzawa. Before performing phenotyping experiments, we first confirmed that Icr1 shows no expression of LCR1 and that this is rescued in the Icr1-C strain (Extended Data Fig. 10a). The Ici8 overexpressing line was purchased from the Chlamydomonas Resource center; strain CSI_FC1G01, expressing pLM005-Cre02.g144800-Venus-3xFLAG in the CC-4533 background. Overexpression of LCI8-FLAG was verified by immunoblotting against FLAG (Extended Data Fig. 10b).

To complement qer1, a 1152 bp genomic DNA fragment from Chlamydomonas CC-4533 was amplified by PCR using KOD hot start DNA polymerase (Merck) and primers P11 and P12 (Supplementary Table 9). To complement qer7, a 5755 bp fragment DNA fragment from Chlamydomonas CC-4533 was amplified by PCR 
with Platinum superfii DNA Polymerase (Thermo Fisher Scientific) and primers P13 and P14 (Supplementary

Table 9). The PCR products were gel purified and cloned into pRAM $118^{68}$ by Gibson assembly ${ }^{69}$ for expression under control of the PSAD promoter. Junctions and insertion were sequenced and constructs were linearized by EcoRV before transformation. Eleven $\mathrm{ng} / \mathrm{kb}$ of linearized plasmid ${ }^{70}$ mixed with $400 \mu \mathrm{L}$ of $1.0 \times 10^{7}$ cells $\mathrm{mL}^{-}$

${ }^{1}$ were electroporated in a volume of $120 \mathrm{~mL}$ in a 2-mm-gap electro cuvette using a NEPA21 square-pulse electroporator (NEPAGENE, Japan). The electroporation parameters were set as follows: Poring Pulse (300V; 8 ms length; 50 ms interval; one pulse; 40\% decay rate; + Polarity), Transfer Pluse (20V; 50 ms length; 50 ms interval; five pulses; $40 \%$ decay rate; +/- Polarity). Transformants were selected onto solid agar plates containing $20 \mu \mathrm{g} / \mathrm{ml}$ hygromycin and screened for fluorescence by using a Tecan fluorescence microplate reader (Tecan Group Ltd., Switzerland). Parameters used were as follows: YFP (excitation 515/12 nm and emission 550/12 nm) and chlorophyll (excitation 440/9 nm and 680/20 nm). Transformants showing high YFP/chlorophyll value were further analyzed by real time qPCR.

Unless otherwise stated, LL conditions corresponded to $15 \mu \mathrm{mol}$ photons $\mathrm{m}^{-2} \mathrm{~s}^{-1}$ while HL conditions corresponded to $300 \mu \mathrm{mol}$ photons $\mathrm{m}^{-2} \mathrm{~s}^{-1}$ of white light (Neptune L.E.D., France; see ${ }^{7}$ for light spectrum). All experiments were repeated at least three times to verify their reproducibility.

\section{DNA Isolation and genotyping of CLiP mutants.}

Total genomic DNA from CLiP mutants and corresponding wild-type strain CC-4533 was extracted according to the protocol suggested by CLiP website (https://www.chlamylibrary.org/). One $\mu$ l of the extracted DNA was used as a template for the PCR assays, using Phire Plant Direct PCR polymerase (Thermo Fisher Scientific). To confirm the CIB1 insertion site in the CLiP mutants, gene-specific primers were used that anneal upstream and downstream of the predicted insertion site of the cassette (primer pairs P3-P4, P7-P8, P9-P10 and P5-P6 for qer6, qer1, qer7 and ger4 respectively; Supplementary Table 9). While all these primers worked in DNA extracted from WT, they did not work in the DNA extracted from the mutants, with the exception of qer4 (Extended Data Fig. 3), therefore primers specific for the 5' and 3' ends of the CIB1

444 Cassette were additionally used. All the primers used for genotyping were shown in Supplementary Table 9. We further confirmed the disruption of the genes of interest by quantifying their mRNA accumulation

(Extended Data Fig. 3).

mRNA quantification 
Bioscience, USA). qPCR reactions were performed and quantitated in a Bio-Rad CFX96 system using SsoAdvanced Universal SYBR Green Supermix (BioRad). The primers $(0.3 \mu \mathrm{M})$ used for qPCR are listed in

Supplementary Table 10. A gene encoding $G$ protein subunit-like protein $(G B L P)^{71}$ was used as the endogenous control , and relative expression values relative to $G B L P$ were calculated from three biological replicates, each of which contained three technical replicates. All primers using for qPCR (Supplementary Table 10) were confirmed as having at least $90 \%$ amplification efficiency. In order to conform mRNA accumulation data to the distributional assumptions of ANOVA, i.e. the residuals should be normally distributed and variances should be equal among groups, Two-Way Analysis of Variance were computed with log-transformed data $\mathrm{Y}=\log \mathrm{X}$ where $\mathrm{X}$ is mRNA accumulation ${ }^{72}$.

\section{Immunoblotting}

Protein samples of whole cell extracts ( $0.5 \mu \mathrm{g}$ chlorophyll or $10 \mu \mathrm{g}$ protein) were loaded on $4-20 \%$ SDS-PAGE gels (Mini-PROTEAN TGX Precast Protein Gels, Bio-Rad) and blotted onto nitrocellulose membranes. Antisera against LHCSR1 (AS14 2819), LHCSR3 (AS14 2766), ATPB (AS05 085), CAH4/5 (AS11 1737) were from Agrisera (Vännäs, Sweden); antiserum against PSBS was from ShineGene Molecular Biotech (Shanghai, China) targeting the peptides described in Ref. ${ }^{9}$. ATPB was used as a loading control. An anti-rabbit horseradish peroxidase-conjugated antiserum was used for detection. The blots were developed with ECL detection reagent, and images of the blots were obtained using a CCD imager (ChemiDoc MP System, Bio-Rad). For the densitometric quantification, data were normalized with ATPB.

\section{Fluorescence-based measurements}

Fluorescence-based photosynthetic parameters were measured with a a pulse modulated amplitude fluorimeter (MAXI-IMAGING-PAM, HeinzWaltz GmbH, Germany). Prior to the onset of the measurements, cells were acclimated to darkness for $15 \mathrm{~min}$. Chlorophyll fluorescence was recorded during 10 min under 570 $\mu \mathrm{mol} \mathrm{m} \mathrm{m}^{-1}$ of actinic blue light followed by finishing with $10 \mathrm{~min}$ of measurements of fluorescence relaxation in the dark. A saturating pulse $(200 \mathrm{msec})$ of blue light $\left(6000 \mu \mathrm{mol}\right.$ photons $\left.\mathrm{m}^{-2} \mathrm{sec}^{-1}\right)$ was applied for determination of Fm (the maximal fluorescence yield in dark-adapted state) or Fm' (maximal fluorescence in of NPQ that is rapidly inducible in the light and reversible in the dark. 
1. Eberhard, S., Finazzi, G. \& Wollman, F. A. The dynamics of photosynthesis. Annu. Rev. Genet. 42, 463-515 (2008).

2. Erickson, E., Wakao, S. \& Niyogi, K. K. Light stress and photoprotection in Chlamydomonas reinhardtii. Plant J. 82, 449-465 (2015).

3. Moroney, J. V. \& Ynalvez, R. A. Proposed carbon dioxide concentrating mechanism in Chlamydomonas reinhardtii. Eukaryotic Cell 6, 1251-1259 (2007).

4. Niyogi, K. K. PHOTOPROTECTION REVISITED: Genetic and Molecular Approaches. Annu. Rev. Plant Physiol. Plant Mol. Biol. 50, 333-359 (1999).

5. Peers, G. et al. An ancient light-harvesting protein is critical for the regulation of algal photosynthesis. Nature 462, 518-521 (2009).

6. Dinc, E. et al. LHCSR1 induces a fast and reversible pH-dependent fluorescence quenching in $\mathrm{LHCll}$ in Chlamydomonas reinhardtii cells. Proc. Natl. Acad. Sci. U.S.A. 113, 7673-7678 (2016).

7. Ruiz-Sola, M. et al. Photoprotection is regulated by light-independent $\mathrm{CO} 2$ availability. bioRxiv (2021). doi:10.1101/2021.10.23.465040

8. Li, X. P. et al. A pigment-binding protein essential for regulation of photosynthetic light harvesting. Nature 403, 391-395 (2000).

9. Correa-Galvis, V. et al. Photosystem II Subunit PsbS Is Involved in the Induction of LHCSR Proteindependent Energy Dissipation in Chlamydomonas reinhardtii. J. Biol. Chem. 291, 17478-17487 (2016).

10. Tibiletti, T., Auroy, P., Peltier, G. \& Caffarri, S. Chlamydomonas reinhardtii PsbS Protein Is Functional and Accumulates Rapidly and Transiently under High Light. Plant Physiol. 171, 2717-2730 (2016).

11. Allorent, G. et al. UV-B photoreceptor-mediated protection of the photosynthetic machinery in Chlamydomonas reinhardtii. Proc. Natl. Acad. Sci. U.S.A. 113, 14864-14869 (2016).

12. Redekop, P. et al. PsbS contributes to photoprotection in Chlamydomonas reinhardtii independently of energy dissipation. BIOCHIMICA ET BIOPHYSICA ACTA-BIOENERGETICS 1861, 148183 (2020).

13. Hanawa, Y., Watanabe, M., Karatsu, Y., Fukuzawa, H. \& Shiraiwa, Y. Induction of a high-CO2inducible, periplasmic protein, $\mathrm{H} 43$, and its application as a high-CO2-responsive marker for study of the high-CO2-sensing mechanism in Chlamydomonas reinhardtii. Plant Cell Physiol. 48, 299-309 (2007).

14. Moroney, J. V. et al. Isolation and Characterization of a Mutant of Chlamydomonas reinhardtii Deficient in the CO2 Concentrating Mechanism. Plant Physiol. 89, 897-903 (1989).

15. Fukuzawa, H. et al. $\mathrm{Ccm} 1$, a regulatory gene controlling the induction of a carbon-concentrating mechanism in Chlamydomonas reinhardtii by sensing $\mathrm{CO} 2$ availability. Proc. Natl. Acad. Sci. U.S.A. 98, 5347-5352 (2001).

16. Xiang, Y., Zhang, J. \& Weeks, D. P. The Cia5 gene controls formation of the carbon concentrating mechanism in Chlamydomonas reinhardtii. Proc. Natl. Acad. Sci. U.S.A. 98, 5341-5346 (2001).

17. Yoshioka, S. et al. The novel Myb transcription factor LCR1 regulates the CO2-responsive gene Cah1, encoding a periplasmic carbonic anhydrase in Chlamydomonas reinhardtii. Plant Cell 16, 1466-1477 (2004).

18. Petroutsos, D. et al. A blue-light photoreceptor mediates the feedback regulation of photosynthesis. Nature 537, 563-566 (2016).

19. Petroutsos, D. et al. The chloroplast calcium sensor CAS is required for photoacclimation in Chlamydomonas reinhardtii. Plant Cell 23, 2950-2963 (2011). 
bioRxiv preprint doi: https://doi.org/10.1101/2022.02.25.482034; this version posted March 2, 2022. The copyright holder for this preprint

(which was not certified by peer review) is the author/funder, who has granted bioRxiv a license to display the preprint in perpetuity. It is made available under aCC-BY-NC-ND 4.0 International license.

524

525

526

527

528

529

530

531

532

533

534

535

536

537

538

539

540

541

542

543

544

545

546

547

548

549

550

551

552

553

554

555

556

557

558

559

560

561

562

563

564

565

566

567

568

569

570

20. Maruyama, S., Tokutsu, R. \& Minagawa, J. Transcriptional regulation of the stress-responsive light harvesting complex genes in Chlamydomonas reinhardtii. Plant Cell Physiol. 55, 1304-1310 (2014).

21. Miura, K. et al. Expression profiling-based identification of CO2-responsive genes regulated by CCM1 controlling a carbon-concentrating mechanism in Chlamydomonas reinhardtii. Plant Physiol. 135, 1595-1607 (2004).

22. Fang, W. et al. Transcriptome-wide changes in Chlamydomonas reinhardtii gene expression regulated by carbon dioxide and the $\mathrm{CO} 2$-concentrating mechanism regulator $\mathrm{CIA} 5 / \mathrm{CCM} 1$. Plant Cell 24, 1876-1893 (2012).

23. Brueggeman, A. J. et al. Activation of the carbon concentrating mechanism by $\mathrm{CO} 2$ deprivation coincides with massive transcriptional restructuring in Chlamydomonas reinhardtii. Plant Cell 24, 1860-1875 (2012).

24. Aihara, Y., Fujimura-Kamada, K., Yamasaki, T. \& Minagawa, J. Algal photoprotection is regulated by the E3 ligase CUL4-DDB1DET1. Nature Plants 5, 34-40 (2019).

25. Redekop, P. et al. Transcriptional regulation of photoprotection in dark-to-light transition- more than just a matter of excess light energy. BioRxiv (2021) https://doi.org/10.1101/2021.10.23.463292

26. Gabilly, S. T. et al. Regulation of photoprotection gene expression in Chlamydomonas by a putative E3 ubiquitin ligase complex and a homolog of CONSTANS. Proc. Natl. Acad. Sci. U.S.A. 116, 1755617562 (2019).

27. Tokutsu, R., Fujimura-Kamada, K., Matsuo, T., Yamasaki, T. \& Minagawa, J. The CONSTANS flowering complex controls the protective response of photosynthesis in the green alga Chlamydomonas. Nat Comms 10, 655-10 (2019).

28. Kamrani, Y. Y., Matsuo, T., Mittag, M. \& Minagawa, J. ROC75 is an Attenuator for the Circadian Clock that Controls LHCSR3 Expression. Plant Cell Physiol. 59, 2602-2607 (2018).

29. Omranian, N., Eloundou-Mbebi, J. M. O., Mueller-Roeber, B. \& Nikoloski, Z. Gene regulatory network inference using fused LASSO on multiple data sets. Sci Rep 6, 1-14 (2016).

30. Marbach, D. et al. Wisdom of crowds for robust gene network inference. Nat. Methods 9, 796-804 (2012).

31. Fang, L. et al. GRNdb: Decoding the gene regulatory networks in diverse human and mouse conditions. Nucl. Acids Res. 49, D97-D103 (2021).

32. Omranian, N. \& Nikoloski, Z. in Methods in Molecular Biology 1629, 283-295 (2017).

33. Huynh-Thu, V. A. \& Sanguinetti, G. in Methods in Molecular Biology 1883, 1-23 (2019).

34. Schäfer, J. \& Strimmer, K. A shrinkage approach to large-scale covariance matrix estimation and implications for functional genomics. Stat Appl Genet Mol Biol 4, Article32 (2005).

35. Zou, H. \& Hastie, T. Regularization and variable selection via the elastic net. Journal of the Royal Statistical Society: Series B (Statistical Methodology) 67, 301-320 (2005).

36. Gargouri, M. et al. Identification of regulatory network hubs that control lipid metabolism in Chlamydomonas reinhardtii. J. Exp. Bot. 66, 4551-4566 (2015).

37. Lämmermann, N. et al. Ubiquitin ligase component LRS1 and transcription factor CrHy5 act as a light switch for photoprotection in \&lt;em\&gt;Chlamydomonas\&lt;/em\&gt. bioRxiv 2020.02.10.942334 (2020).

38. Zones, J. M., Blaby, I. K., Merchant, S. S. \& Umen, J. G. High-Resolution Profiling of a Synchronized Diurnal Transcriptome from Chlamydomonas reinhardtii Reveals Continuous Cell and Metabolic Differentiation. Plant Cell 27, 2743-2769 (2015).

39. Strenkert, D. et al. Multiomics resolution of molecular events during a day in the life of Chlamydomonas. Proc. Natl. Acad. Sci. U.S.A. 116, 2374-2383 (2019).

40. Fett, J. P. \& Coleman, J. R. Regulation of Periplasmic Carbonic Anhydrase Expression in Chlamydomonas reinhardtii by Acetate and pH. Plant Physiol. 106, 103-108 (1994). 
bioRxiv preprint doi: https://doi.org/10.1101/2022.02.25.482034; this version posted March 2, 2022. The copyright holder for this preprint

(which was not certified by peer review) is the author/funder, who has granted bioRxiv a license to display the preprint in perpetuity. It is made available under aCC-BY-NC-ND 4.0 International license.

571 41. Pérez-Rodríguez, P. et al. PInTFDB: Updated content and new features of the plant transcription

42. Jin, J. et al. PlantTFDB 4.0: Toward a central hub for transcription factors and regulatory interactions in plants. Nucl. Acids Res. 45, D1040-D1045 (2017).

43. Fischer, B. B. et al. SINGLET OXYGEN RESISTANT 1 links reactive electrophile signaling to singlet oxygen acclimation in Chlamydomonas reinhardtii. Proc. Natl. Acad. Sci. U.S.A. 109, E1302-11 (2012).

44. Margolin, A. A. et al. ARACNE: An algorithm for the reconstruction of gene regulatory networks in a mammalian cellular context. BMC Bioinformatics 7, 1-15 (2006).

45. Barzel, B. \& Barabási, A.-L. Network link prediction by global silencing of indirect correlations. Nature Biotechnology 31, 720-725 (2013).

46. Omranian, N., Eloundou-Mbebi, J. M. O., Mueller-Roeber, B. \& Nikoloski, Z. Gene regulatory network inference using fused LASSO on multiple data sets. Sci Rep 6, 20533 (2016).

47. de Borda, J.-C. Mémoire sur les élections au scrutin. Histoire de IAcademie Royale des Sciences (1781).

48. Li, X. et al. A genome-wide algal mutant library and functional screen identifies genes required for eukaryotic photosynthesis. Nat. Genet. 51, 627-635 (2019).

49. UniProt Consortium. UniProt: the universal protein knowledgebase in 2021. Nucleic Acids Res. 49, D480-D489 (2021).

50. Mi, H. et al. PANTHER version 16: A revised family classification, tree-based classification tool, enhancer regions and extensive API. Nucl. Acids Res. 49, D394-D403 (2021).

51. Thiriet-Rupert, S. et al. Transcription factors in microalgae: genome-wide prediction and comparative analysis. BMC Genomics 17, 282 (2016).

52. Bushnell, B. BBMap: A Fast, Accurate, Splice-Aware Aligner. Lawrence Berkeley National Laboratory. LBNL Report LBNL-E (2014).

53. Law, C. W., Chen, Y., Shi, W. \& Smyth, G. K. Voom: Precision weights unlock linear model analysis tools for RNA-seq read counts. Genome Biology 15, 1-17 (2014).

54. Anders, S., Pyl, P. T. \& Huber, W. HTSeq-A Python framework to work with high-throughput sequencing data. Bioinformatics 31, 166-169 (2015).

55. Robinson, M. D. \& Oshlack, A. A scaling normalization method for differential expression analysis of RNA-seq data. Genome Biology 11, 1-9 (2010).

56. Robinson, M. D., McCarthy, D. J. \& Smyth, G. K. edgeR: A Bioconductor package for differential expression analysis of digital gene expression data. Bioinformatics 26, 139-140 (2009).

57. Huynh-Thu, V. A., Irrthum, A., Wehenkel, L. \& Geurts, P. Inferring regulatory networks from expression data using tree-based methods. PLOS ONE 5, e12776 (2010).

58. Meyer, P. E., Lafitte, F. \& Bontempi, G. Minet: A r/bioconductor package for inferring large transcriptional networks using mutual information. BMC Bioinformatics 9, 1-10 (2008).

59. Olsen, C., Meyer, P. E. \& Bontempi, G. On the impact of entropy estimation on transcriptional regulatory network inference based on mutual information. Eurasip Journal on Bioinformatics and Systems Biology 2009, (2009).

60. Faith, J. J. et al. Large-scale mapping and validation of Escherichia coli transcriptional regulation from a compendium of expression profiles. PLoS Biol. 5, 0054-0066 (2007).

61. Feizi, S., Marbach, D., Médard, M. \& Kellis, M. Network deconvolution as a general method to distinguish direct dependencies in networks. Nature Biotechnology 31, 726-733 (2013).

62. Ritchie, M. E. et al. Limma powers differential expression analyses for RNA-sequencing and microarray studies. Nucl. Acids Res. 43, e47 (2015).

63. Love, M. I., Huber, W. \& Anders, S. Moderated estimation of fold change and dispersion for RNA-seq data with DESeq2. Genome Biology 15, 1-21 (2014). 
64. North, B. V., Curtis, D. \& Sham, P. C. A note on the calculation of empirical P values from Monte Carlo procedures [1]. American Journal of Human Genetics 71, 439-441 (2002).

65. Gorman, D. S. \& Levine, R. P. Cytochrome $f$ and plastocyanin: their sequence in the photosynthetic electron transport chain of Chlamydomonas reinhardii. Proc. Natl. Acad. Sci. U.S.A. 54, 1665-1669 (1965).

66. Sueoka, N. Mitotic replication of deoxyribonucleic acid in Chlamydomonas reinhardii. Proc. Natl. Acad. Sci. U.S.A. 46, 83-91 (1960).

67. Greiner, A. et al. Targeting of Photoreceptor Genes in Chlamydomonas reinhardtii via Zinc-Finger Nucleases and CRISPR/Cas9. Plant Cell 29, 2498-2518 (2017).

68. Kaye, Y. et al. The mitochondrial alternative oxidase from Chlamydomonas reinhardtii enables survival in high light. J. Biol. Chem. 294, 1380-1395 (2019).

69. Gibson, D. G. et al. Enzymatic assembly of DNA molecules up to several hundred kilobases. Nat. Methods 6, 343-345 (2009).

70. Mackinder, L. C. M. et al. A repeat protein links Rubisco to form the eukaryotic carbon-concentrating organelle. Proc. Natl. Acad. Sci. U.S.A. 113, 5958-5963 (2016).

71. Schloss, J. A. A Chlamydomonas gene encodes a G protein beta subunit-like polypeptide. Molec. Gen. Genet. 221, 443-452 (1990).

72. Schlesselman, J. J. Data Transformation in Two-Way Analysis of Variance. Journal of the American Statistical Association (2012). doi:10.1080/01621459.1973.10482435

73. Genty, B., Briantais, J.-M. \& Baker, N. R. The relationship between the quantum yield of photosynthetic electron transport and quenching of chlorophyll fluorescence. Biochimica et Biophysica Acta (BBA) 990, 87-92 (1989). 


\section{Acknowledgments}

643 We are grateful to Prof. Hideya Fukuzawa for sending us the $l c r l$ and $l c r l-C$ strains and to Prof. Peter 644 Jahns for the antibody against PSBS

645

646 Funding:

647 The authors would like to thank the following agencies for funding: The Human Frontiers Science 648 Program through the funding of the project RGP0046/2018 (DP, ZN); the French National Research 649 Agency in the framework of the Young Investigators program ANR-18-CE20-0006 through the 650 funding of the project MetaboLight (DP); the French National Research Agency in the framework of 651 the Investissements d'Avenir program ANR-15-IDEX-02, through the funding of the "Origin of Life" 652 project of the Univ. Grenoble-Alpes (DP, YY); the French National Research Agency through the 653 funding of the Grenoble Alliance for Integrated Structural \& Cell Biology GRAL project ANR-17654 EURE-0003 (DP, MAR-S), the Prestige Marie-Curie co-financing grant PRESTIGE-2017-1-0028 655 (MAR-S); the International Max Planck Research School 'Primary Metabolism and Plant Growth' at 656 the Max Planck Institute of Molecular Plant Physiology (MA, ZN).

Competing interests: Authors declare that they have no competing interests. 
Fig. 1: Characterization of the consensus GRN inferred by employing a compendium of RNA-seq data from diverse light and culture conditions. a. Expression levels of representative CCM and $\mathrm{qE}$ related genes are plotted over all samples used for network inference (z-scaled log values are depicted). The column annotation gives information on the culture conditions. The values of the rows of the heatmap correspond to the z-scores of representative CCM and qE genes plotted over all samples (columns) used in the network inference. b. The heatmap rows correspond to experimentally validated or falsified (neg) gene regulatory interactions involved in $\mathrm{qE}$ and CCM, curated from literature. The heatmap indicates ranking of these interactions by different approaches and the consensus network. Edges are considered highly ranked (depicted in blue) if they are above the $10 \%$ network density threshold. Edges ranked below this threshold are depicted in red. Edges that were not included in the given network are marked in grey. ARACNE and Silencing columns were only plotted for comparison and were not used in building the consensus GRN (see Methods).

Fig. 2: Consensus GRN for light and acetate responses pinpoints LCR1 as regulator of qE-related genes. a. Dot plot of the relative regulatory strength of the top 10 regulators of $q E$-related genes in the consensus GRN (see Methods). TFs are marked in green if qE transcript levels were affected in the respective knock-out strain and this effect was reversed by complementation with the missing gene. TFs for which no effect was observed are marked in red. TFs for which no mutant lines were available are plotted in grey. $\mathbf{b}$. WT, Icr1 and Icr1-C cells were acclimated for $16 \mathrm{~h}$ in $\mathrm{LL}\left(15 \mu \mathrm{mol}\right.$ photons $\left.\mathrm{m}^{-2} \mathrm{~s}^{-1}\right)$. After sampling for the LL conditions, light intensity was increased to $300 \mu \mathrm{mol}$ photons $\mathrm{m}^{-2} \mathrm{~s}^{-1}(\mathrm{HL})$; samples were taken $1 \mathrm{~h}$ (RNA) or $4 \mathrm{~h}$ (protein and photosynthetic measurements) after exposure to HL. Shown are relative expression levels of qE-related genes at the indicated conditions normalized to WT LL ( $n=3$ biological samples, mean $\pm \mathrm{sd}$ ). c. Immunoblot analyses of LHCSR1, LHCSR3, PSBS and ATPB (loading control) of one of the three biological replicates, under the indicated conditions. d. Quantification of immunoblot data of all replicates in panel $\mathbf{c}$ after normalization to ATPB. Shown are the HL treated samples; WT protein levels were set as 1. e. NPQ and calculated qE (as an inset) $4 \mathrm{~h}$ after exposure to HL ( $n=3$ biological samples, mean \pm s.d). The $p$-values for the comparisons are based on ANOVA Dunnett's multiple comparisons test and as indicated in the graphs $\left({ }^{*}, \mathrm{P}<\right.$ $0.005, * *, \mathrm{P}<0.01, * * *, \mathrm{P}<0.001, * * * *, \mathrm{P}<0.0001)$. Statistical analyses for panel $\mathbf{b}$ and $\mathbf{d}$ were applied on log10- transformed values.

Fig. 3: A PHOT-specific GRN pinpoints QER7 as a suppressor of the expression of qE-related genes. a. Dot plot of the relative regulatory strength of the top 10 regulators of $q E$-related genes in the PHOT-specific GRN (see Methods). TFs are marked in green if qE transcript levels were affected in the respective knock-out strain and this effect was reversed by complementation with the knocked-out gene, in yellow, if the effect was not reversed by complementation, and in red, if no mutant effect was observed in the mutant. TFs for which no mutant lines were available are plotted in grey. b. WT, qer7 and ger7-C cells were synchronized under $12 \mathrm{~h}$ light $\left(15 \mu \mathrm{mol} \mathrm{m} \mathrm{m}^{-2} \mathrm{~s}^{-1}\right) / 12 \mathrm{~h}$ dark cycles. After sampling for the dark conditions (end of the dark phase), cells were exposed to $300 \mu \mathrm{mol}$ photons $\mathrm{m}^{-2} \mathrm{~s}^{-1}(\mathrm{HL})$; samples were taken $1 \mathrm{~h}$ (RNA) or $4 \mathrm{~h}$ (protein and photosynthetic measurements) after exposure to HL. Shown are relative expression levels of qE-related genes at the indicated conditions normalized to WT LL $(n=3$ 
biological samples, mean $\pm \mathrm{sd}$ ). c. Immunoblot analyses of LHCSR1, LHCSR3 and ATPB (loading control) of one of the three biological replicate set of samples, under the indicated conditions. d. Quantification of immunoblot data of all replicates in panel $\mathbf{c}$ after normalization to ATPB. WT protein levels at $\mathrm{HL}$ were set as 1. e. NPQ and $\mathrm{qE}$, measured $4 \mathrm{~h}$ after exposure to $\mathrm{HL}(n=3$ biological samples, mean $\pm \mathrm{sd}$ ). The $p$-values for the comparisons are based on ANOVA Dunnett's multiple comparisons test and are indicated in the graphs $\left({ }^{*}, \mathrm{P}<0.005, * *, \mathrm{P}<0.01\right.$, $* * *, \mathrm{P}<0.001, * * * *, \mathrm{P}<0.0001)$. Statistical analyses for panel $\mathbf{b}$ and $\mathbf{d}$ were applied on $\log 10-$ transformed values.

Fig:4: QER7 suppresses transcription of CCM genes and depends on PHOT. WT, qer7 and qer7-C cells were synchronized under $12 \mathrm{~h}$ light $\left(15 \mu \mathrm{mol} \mathrm{m}^{-2} \mathrm{~s}^{-1}\right) / 12 \mathrm{~h}$ dark cycles. After sampling at the end of the dark phase, cells were exposed to $300 \mu \mathrm{mol}$ photons $\mathrm{m}^{-2} \mathrm{~s}^{-1}(\mathrm{HL})$ and samples were taken $1 \mathrm{~h}$ after $\mathrm{HL}$ exposure. a. Relative expression levels of CCM genes at the indicated conditions normalized to WT LL ( $n=3$ biological samples, mean $\pm s d$ ). $\mathbf{b}$ and $\mathbf{c}$. Relative expression levels of $Q E R 7$ (b) and LCR1 (c) in synchronized WT, phot and phot-C (phot cells complemented with the PHOT gene) cells. The $p$-values for the comparisons are based on ANOVA Dunnett's multiple comparisons test on log10- transformed values and are indicated in the graphs $\left(^{*}, \mathrm{P}<0.005\right.$, $* *, \mathrm{P}<0.01, * * *, \mathrm{P}<0.001, * * * *, \mathrm{P}<0.0001)$.

Fig. 5: Consensus and PHOT-specific GRNs indicate extensive co-regulation of CCM and photoprotective genes. Top: Venn diagram depicting the overlap of the top 10 predicted TFs of the curate CCM and photoprotective ( $\mathrm{PhPr}$ ) genes based on a. the consensus or c. PHOTspecific GRN. Bottom: Network representation of the top 10 TF sets of $\mathbf{b}$. the consensus network or $\mathbf{d}$. the phot GRN (center nodes, same color code as in panel a and the target genes used for prediction (left and right columns of nodes, photoprotection genes are shown in green, qE-related genes in blue, and CCM genes in red. Larger TF nodes indicate higher regulatory strength on target genes. The plotted edge width is proportional to the strength of the regulatory interaction. 
bioRxiv preprint doi: https://doi.org/10.1101/2022.02.25.482034; this version posted March 2, 2022. The copyright holder for this preprint (which was not certified by peer review) is the author/funder, who has granted bioRxiv a license to display the preprint in perpetuity. It is made available under aCC-BY-NC-ND 4.0 International license.

a

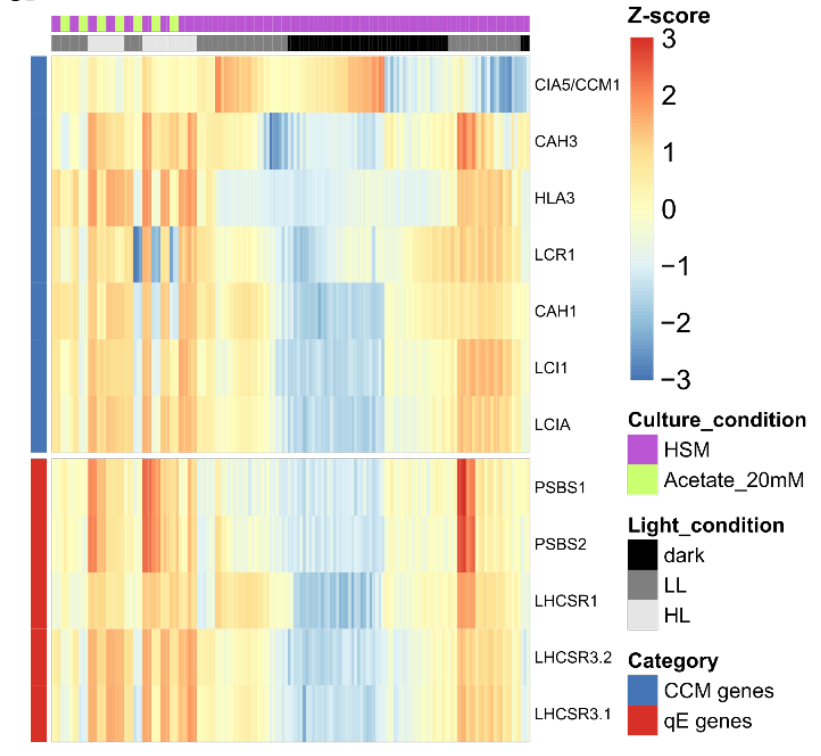

b

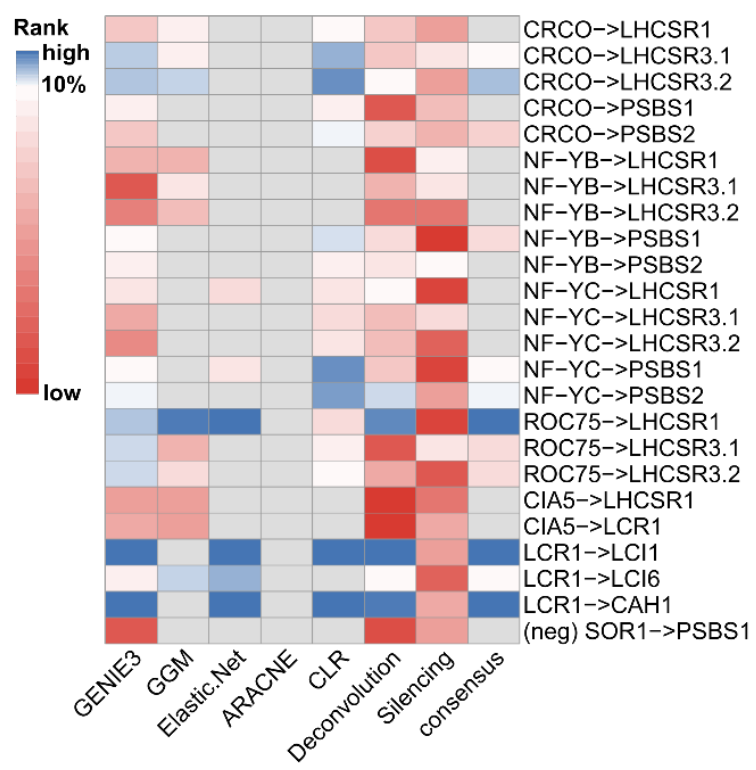

Fig. 1: Characterization of the consensus GRN inferred by employing a compendium of RNA-seq data from diverse light and culture conditions. a. Expression levels of representative CCM and qE related genes are plotted over all samples used for network inference (z-scaled log values are depicted). The column annotation gives information on the culture conditions. The values of the rows of the heatmap correspond to the z-scores of representative CCM and qE genes plotted over all samples (columns) used in the network inference. $\mathbf{b}$. The heatmap rows correspond to experimentally validated or falsified (neg) gene regulatory interactions involved in $\mathrm{qE}$ and $\mathrm{CCM}$, curated from literature. The heatmap indicates ranking of these interactions by different approaches and the consensus network. Edges are considered highly ranked (depicted in blue) if they are above the $10 \%$ network density threshold. Edges ranked below this threshold are depicted in red. Edges that were not included in the given network are marked in grey. ARACNE and Silencing columns were only plotted for comparison and were not used in building the consensus GRN (see Methods). 
a

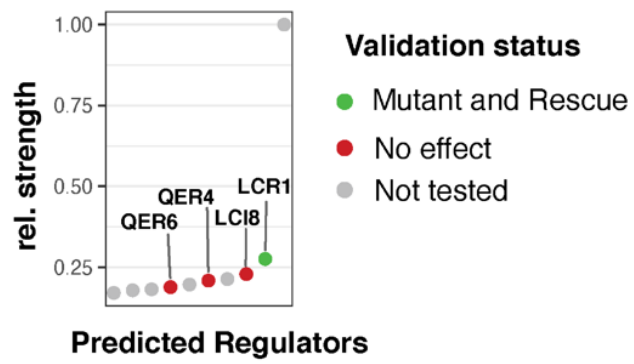

b

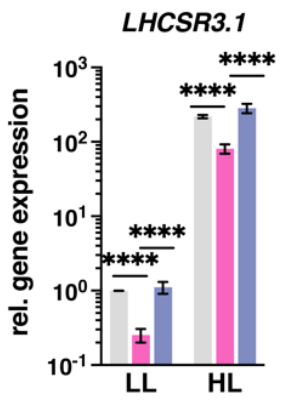

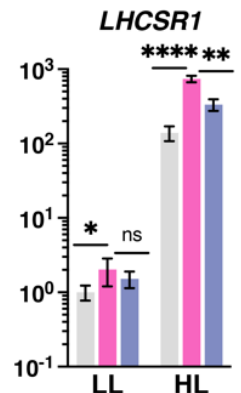

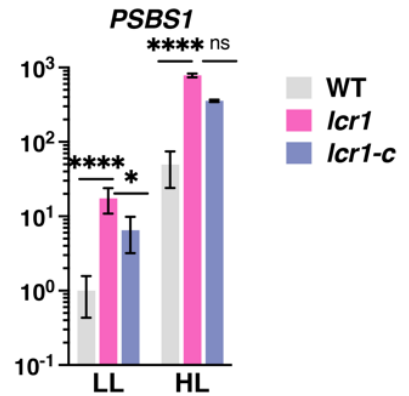

747

C

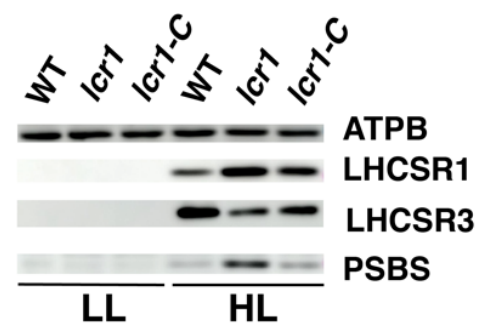

d

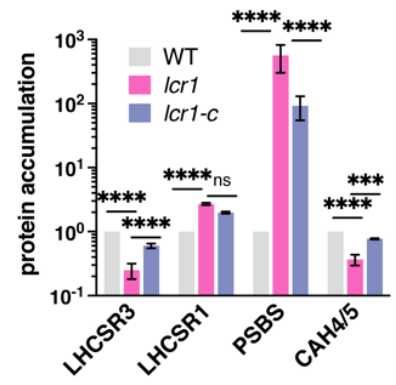

e

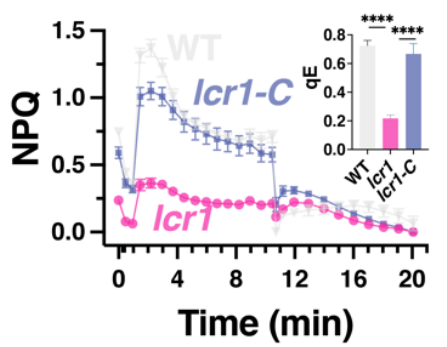

748

749

750

751

752

753

754

755

756

757

758

759

760

761

762

763

764

765

766

767

Fig. 2: Consensus GRN for light and acetate responses pinpoints LCR1 as regulator of qE-related genes. a. Dot plot of the relative regulatory strength of the top 10 regulators of qE-related genes in the consensus GRN (see Methods). TFs are marked in green if qE transcript levels were affected in the respective knock-out strain and this effect was reversed by complementation with the missing gene. TFs for which no effect was observed are marked in red. TFs for which no mutant lines were available are plotted in grey. b. WT, Icr1 and Icr1-C cells were acclimated for $16 \mathrm{~h}$ in LL $\left(15 \mu \mathrm{mol}\right.$ photons $\left.\mathrm{m}^{-2} \mathrm{~s}^{-1}\right)$. After sampling for the LL conditions, light intensity was increased to $300 \mu \mathrm{mol}$ photons $\mathrm{m}^{-2} \mathrm{~s}^{-1}(\mathrm{HL})$; samples were taken $1 \mathrm{~h}$ (RNA) or $4 \mathrm{~h}$ (protein and photosynthetic measurements) after exposure to $\mathrm{HL}$. Shown are relative expression levels of qE-related genes at the indicated conditions normalized to WT LL ( $n=3$ biological samples, mean $\pm s d)$. c. Immunoblot analyses of LHCSR1, LHCSR3, PSBS and ATPB (loading control) of one of the three biological replicates, under the indicated conditions. d. Quantification of immunoblot data of all replicates in panel $\mathbf{c}$ after normalization to ATPB. Shown are the HL treated samples; WT protein levels were set as 1. e. NPQ and calculated qE (as an inset) $4 \mathrm{~h}$ after exposure to $\mathrm{HL}(n=3$ biological samples, mean $\pm \mathrm{s}$.d). The $\mathrm{p}$-values for the comparisons are based on ANOVA Dunnett's multiple comparisons test and as indicated in the graphs $(*, \mathrm{P}<0.005, * *, \mathrm{P}<$ $0.01, * * *, \mathrm{P}<0.001, * * * *, \mathrm{P}<0.0001)$. Statistical analyses for panel $\mathbf{b}$ and $\mathbf{d}$ were applied on $\log 10-$ transformed values. . 
a

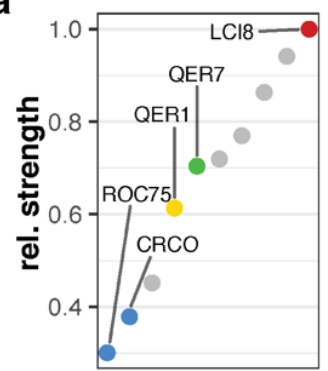

Validation status

- Mutant

- Mutant and Rescue

- No effect

- Reported

- Not tested b

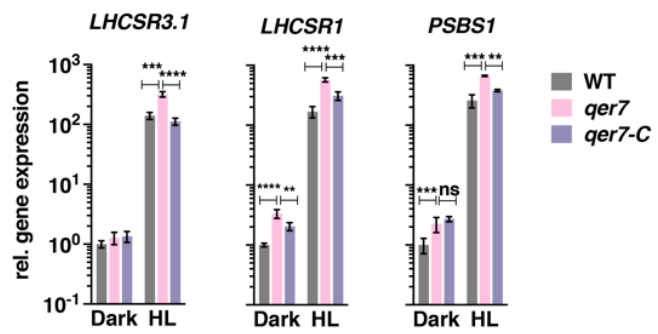

Predicted Regulators

C

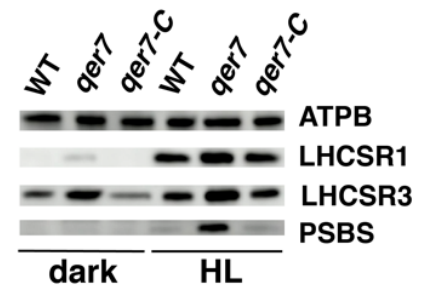

d

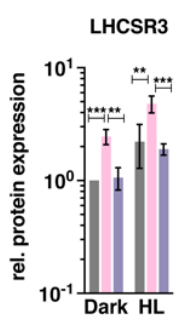

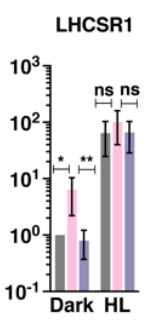

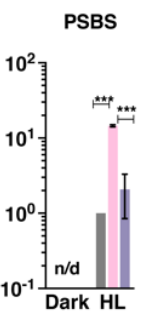

e

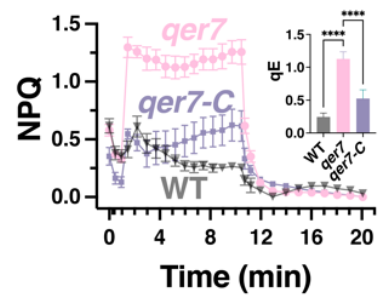

Fig. 3: A PHOT-specific GRN pinpoints QER7 as a suppressor of the expression of qE-related genes. a. Dot plot of the relative regulatory strength of the top 10 regulators of qE-related genes in the PHOT-specific GRN (see Methods). TFs are marked in green if qE transcript levels were affected in the respective knock-out strain and this effect was reversed by complementation with the knocked-out gene, in yellow, if the effect was not reversed by complementation, and in red, if no mutant effect was observed in the mutant. TFs for which no mutant lines were available are plotted in grey. b. WT, qer7 and ger7-C cells were synchronized under $12 \mathrm{~h}$ light $\left(15 \mu \mathrm{mol} \mathrm{m} \mathrm{m}^{-2} \mathrm{~s}^{-1}\right) / 12 \mathrm{~h}$ dark cycles. After sampling for the dark conditions (end of the dark phase), cells were exposed to $300 \mu \mathrm{mol}$ photons $\mathrm{m}^{-2} \mathrm{~s}^{-1}(\mathrm{HL})$; samples were taken $1 \mathrm{~h}$ (RNA) or $4 \mathrm{~h}$ (protein and photosynthetic measurements) after exposure to $\mathrm{HL}$. Shown are relative expression levels of qE-related genes at the indicated conditions normalized to WT LL ( $n=3$ biological samples, mean $\pm s d)$. c. Immunoblot analyses of LHCSR1, LHCSR3 and ATPB (loading control) of one of the three biological replicate set of samples, under the indicated conditions. d. Quantification of immunoblot data of all replicates in panel $\mathbf{c}$ after normalization to ATPB. WT protein levels at HL were set as 1 . e. NPQ and qE, measured 4 h after exposure to $H L(n=3$ biological samples, mean $\pm \mathrm{sd}$ ). The $\mathrm{p}$-values for the comparisons are based on ANOVA Dunnett's multiple comparisons test and are indicated in the graphs $(*, \mathrm{P}<0.005, * *, \mathrm{P}<0.01, * * *, \mathrm{P}<0.001, * * * *, \mathrm{P}<0.0001)$. 
a
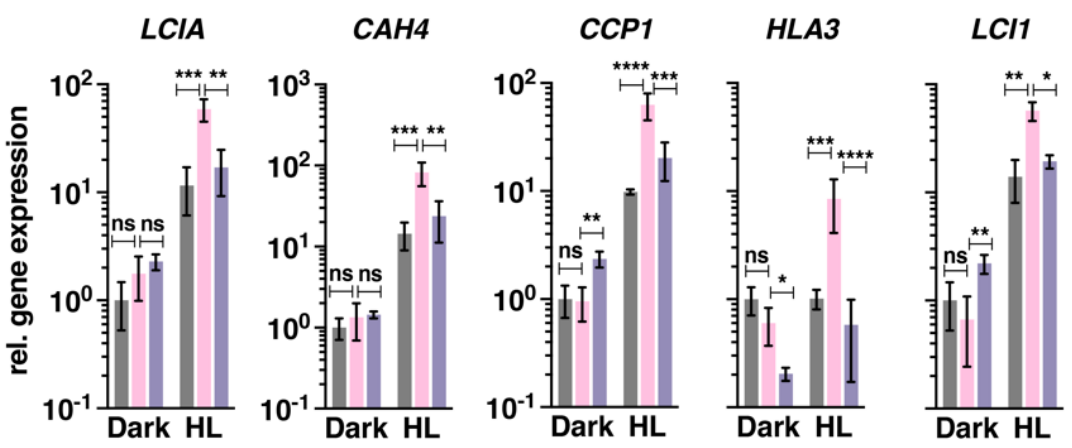

BST1

b

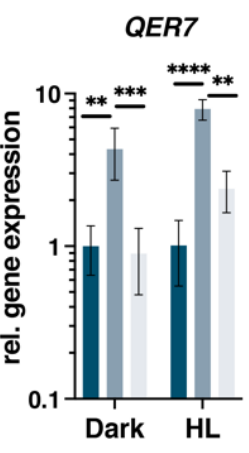

C LCR1

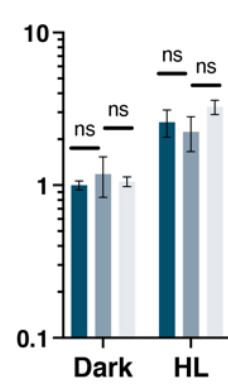

WT

phot

phot-C

Fig:4: QER7 suppresses transcription of CCM genes and depends on PHOT. WT, ger7 and qer7-C cells were synchronized under $12 \mathrm{~h}$ light $\left(15 \mu \mathrm{mol} \mathrm{m} \mathrm{m}^{-2} \mathrm{~s}^{-1}\right) / 12 \mathrm{~h}$ dark cycles. After sampling at the end of the dark phase, cells were exposed to $300 \mu \mathrm{mol}$ photons $\mathrm{m}^{-2} \mathrm{~s}^{-1}(\mathrm{HL})$ and samples were taken $1 \mathrm{~h}$ after $\mathrm{HL}$ exposure. a. Relative expression levels of CCM genes at the indicated conditions normalized to WT LL ( $n=3$ biological samples, mean $\pm \mathrm{sd}$ ). $\mathbf{b}$ and $\mathbf{c}$. Relative expression levels of $Q E R 7$ (b) and $L C R 1$ (c) in synchronized WT, phot and phot-C (phot cells complemented with the PHOT gene) cells. The p-values for the comparisons are based on ANOVA Dunnett's multiple comparisons test on log10- transformed values and are indicated in the graphs $\left(^{*}, \mathrm{P}<\right.$ 
a

b

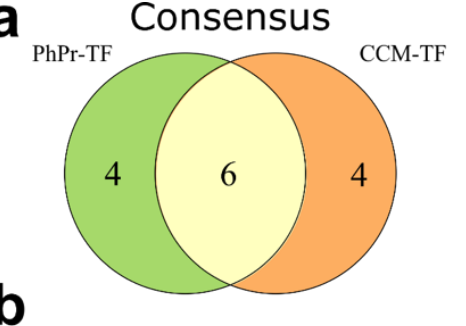

PhPr-

Genes

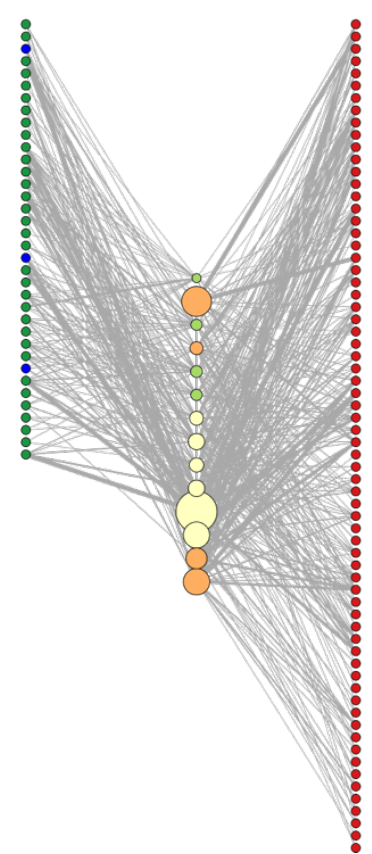

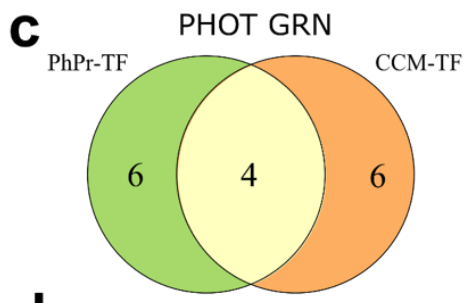

d

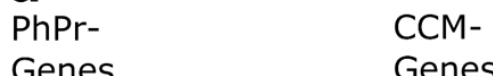

798

799

800

801

802

803

804

805

806

Fig. 5: Consensus and PHOT-specific GRNs indicate extensive co-regulation of CCM and photoprotective genes. Top: Venn diagram depicting the overlap of the top 10 predicted TFs of the curate CCM and photoprotective $(\mathrm{PhPr})$ genes based on a. the consensus or c. PHOT-specific GRN. Bottom: Network representation of the top 10 TF sets of $\mathbf{b}$. the consensus network or $\mathbf{d}$. the phot GRN (center nodes, same color code as in panel $\mathbf{a}$ and the target genes used for prediction (left and right columns of nodes, photoprotection genes are shown in green, qE-related genes in blue, and CCM genes in red. Larger TF nodes indicate higher regulatory strength on target genes. The plotted edge width is proportional to the strength of the regulatory interaction.

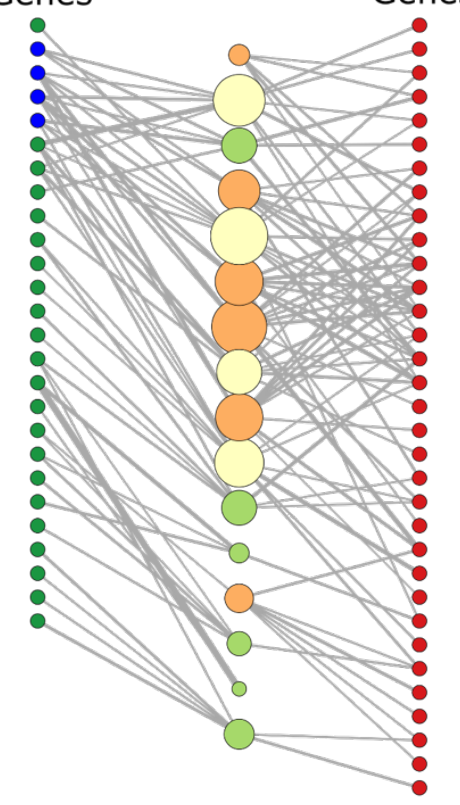


3 Widening the landscape of transcriptional regulation of algal photoprotection

4

\section{Authors}

Marius Arend ${ }^{1,2+}$, Yizhong Yuan ${ }^{3 \dagger}$, M. Águila Ruiz-Sola ${ }^{3 \ddagger}$, Nooshin Omranian ${ }^{1,2,4}$, Zoran Nikoloski ${ }^{1,2,4^{*}}$, Dimitris 7 Petroutsos $^{3 *}$

$9{ }^{\dagger}$ these authors contributed equally to this work

*Corresponding Authors: nikoloski@mpimp-golm.mpg.de (Z.N.), dimitris.petroutsos@cea.fr (D.P.)

12

13

This PDF file includes:

Supplementary Note

Extended Data Figs. 1 to 10

Other Supplementary Materials for this manuscript include the following:

Tables S1 to S10 (to be downloaded as excel files) 


\section{Supplementary Note}

31 Simulation studies ${ }^{30}$ indicated that the top $10 \%$ of the edges in the consensus network are enriched in positive interactions if the underlying approaches perform better than guessing. Thus, a higher overlap with the consensus networks is expected to result in improved predictions. We found that the interactions from the network deconvolution approach exhibited the largest overlap with the consensus network, while GGM assigns the highest rank to consensus interactions (Extended Data Fig. 2a). Repeating this analysis by including the two worse performing approaches (i.e. ARACNE and Silencing) resulted in no qualitative changes in the overlaps (Extended Data Fig. 2b) and only $7.47 \%$ of difference to the original consensus network, demonstrating the robustness of the inferred interactions. Considering the $10 \%$ network density threshold, we also inspected the proportion of TF-TF interactions in the different networks and their consensus; we found that it ranges from $2.55 \%$ in the GENIE3 network to $2.77 \%$ in the network deconvolution approach interactions has a relative TF-TF content of $2.52 \%$, these findings suggest an enrichment of TF-TF interactions in GRNs inferred by all five approaches considered in the consensus. 
bioRxiv preprint doi: https://doi.org/10.1101/2022.02.25.482034; this version posted March 2, 2022. The copyright holder for this preprint (which was not certified by peer review) is the author/funder, who has granted bioRxiv a license to display the preprint in perpetuity. It is made available under aCC-BY-NC-ND 4.0 International license.

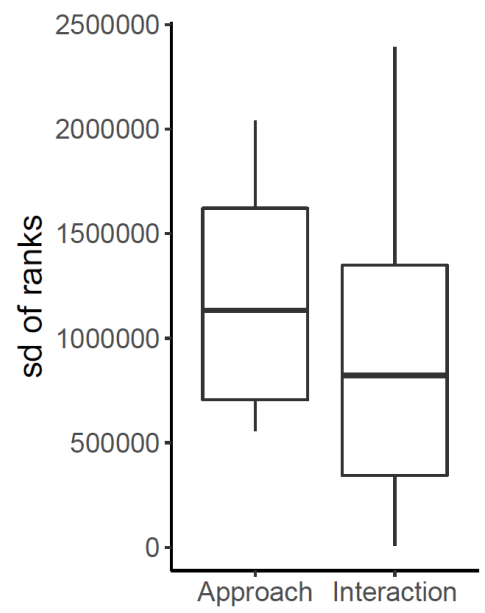

47

48 Extended Data Fig. 1: Variation in ranks of known interactions across approaches and interactions from 49 different inference approaches. Boxplots of the standard deviation of ranks calculated for the curated 50 interactions plotted in Fig. 1b over all ranks assigned by one approach or over all ranks assigned to one 51 interaction.

52 
a

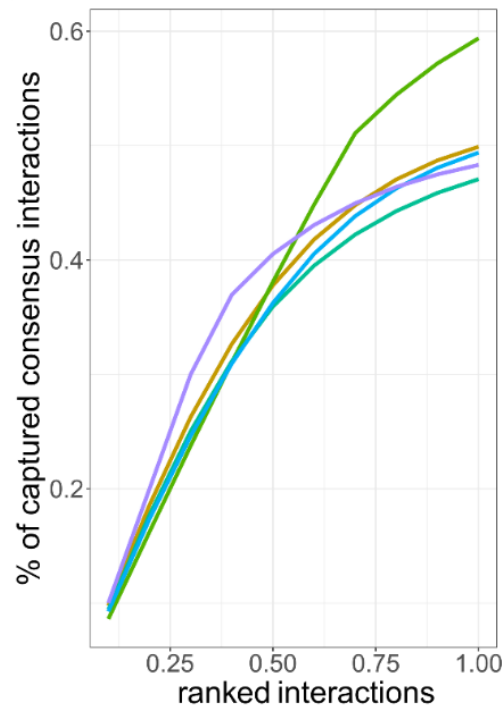

b

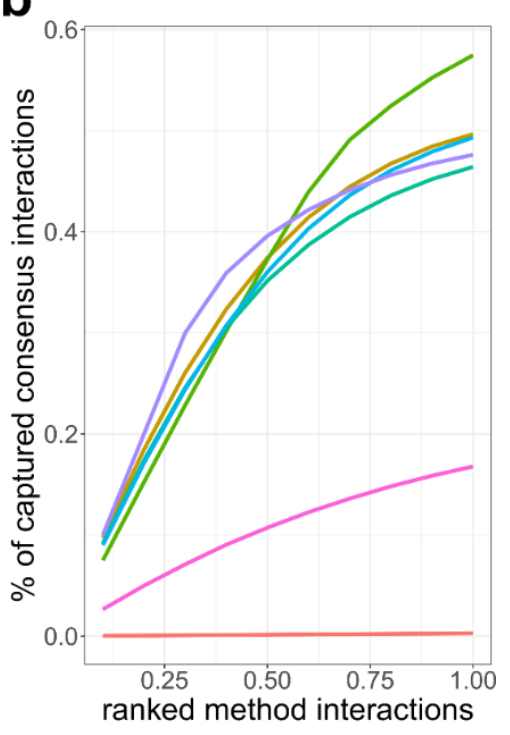

C

method

- ARACNE

- Deconvolution

- Elastic Net

- GENIE3

- GGM

- Silencing

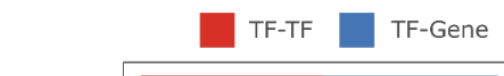

Extended Data Fig. 2: Overlap of consensus network with individual inference methods and proportion of

TF-TF interactions in the obtained GRNs. a. The graph shows the overlap between the edges of the consensus network and the ranked interactions of the individual approaches normalized to the total number of edges in the consensus network used for regulator prediction. b. Same as in a but plotting the overlap with a consensus of all used approaches (including ARACNE and Silencing). c. Bar plots provide the proportion of TF-TF interactions in comparison to TF-target gene interactions contained in the GRNs inferred by the different approaches and the consensus GRN resulting from their integration. All depicted analyses considered only the interactions within in the $10 \%$ network density threshold (see Methods). 
a
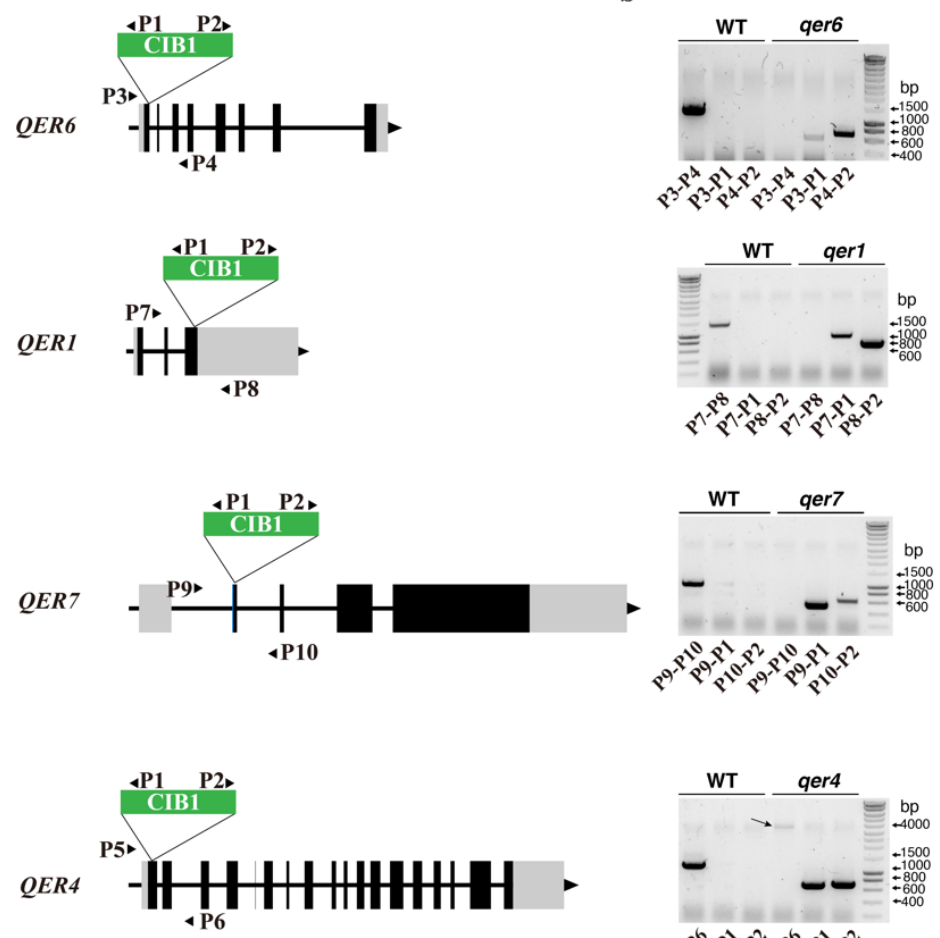

$1 \mathrm{~Kb}$ b
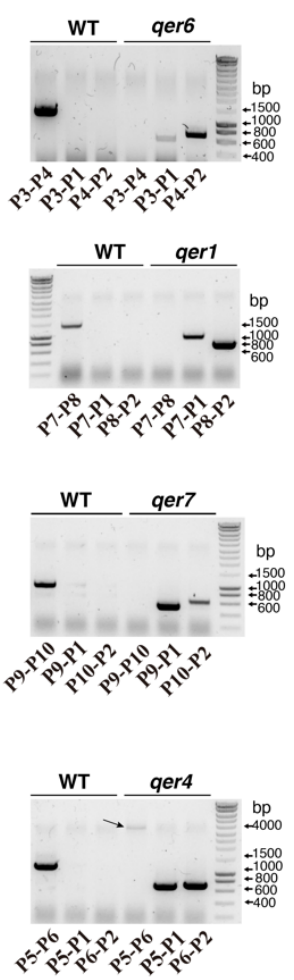

c
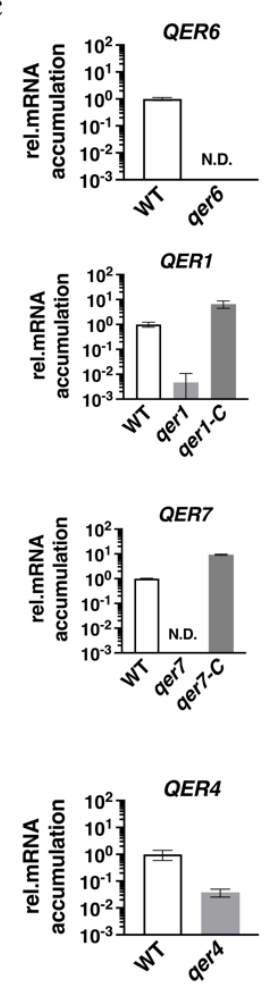

Extended Data Fig. 3: Genotyping of CLiP mutants affected in the predicted regulators of qE-related genes. a. Insertion map of the CIB1 cassette in the different genes. Exons are shown in black, introns as interconnecting lines, 5'UTR and 3'UTR in light gray, and primers in arrows. The insertion site of the CIB1 cassette is indicated by the triangle; $\mathbf{b}$. PCR-validation of the insertion site in the different CLiP mutants using genomic DNA. To confirm the CIB1 insertion site, gene-specific primers were used that anneal upstream and downstream of the predicted insertion site of the cassette (primer pairs P3-P4, P7-P8, P9-P10 and P5-P6 for qer6, qer1, qer7 and qer4 respectively; Supplementary Table 9). Pairs of primers used are indicated at the bottom of the agarose gels used to separate the PCR products. Note that the PCR product of P5-P6 is indicated by an arrow. c. Relative expression levels of predicted $q E$ regulator genes in the different CLiP mutants after exposure to $\mathrm{HL}\left(300 \mu \mathrm{mol}\right.$ photons $\left.\mathrm{m}^{-2} \mathrm{~s}^{-1}\right)$ for $1 \mathrm{~h}$. 
bioRxiv preprint dol: https://doi.org/10.1101/2022.02.25.482034; this version posted March 2, 2022. The copyright holder for this preprint (which was not certified by peer review) is the author/funder, who has granted bioRxiv a license to display the preprint in perpetuity. It is made available under aCC-BY-NC-ND 4.0 International license.

LHCSR3.1

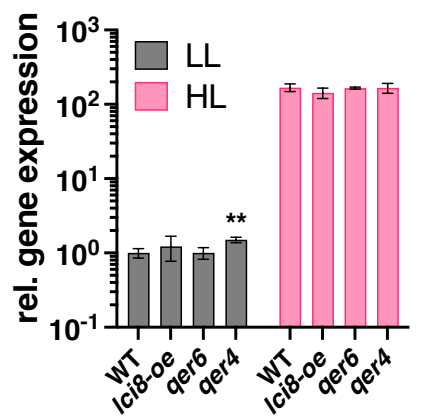

LHCSR1

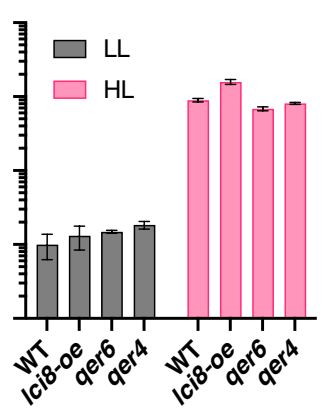

PSBS1

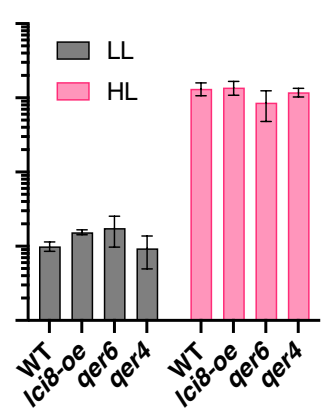

80

Extended Data Fig. 4: pE gene and protein expression in CLiP mutants bearing mutations in predicted pE regulator genes. WT, Ici8-oe, ger and ger cells were acclimated for $16 \mathrm{~h}$ in $\mathrm{LL}\left(15 \mu \mathrm{mol}\right.$ photons $\left.\mathrm{m}^{-2} \mathrm{~s}^{-1}\right)$. After sampling for the LL conditions, light intensity was increased to $300 \mu \mathrm{mol}$ photons $\mathrm{m}^{-2} \mathrm{~s}^{-1}(\mathrm{HL})$; samples were taken $1 \mathrm{~h}$ after exposure to HL. Shown are relative expression levels of of LHCSR3.1, LHCSR1 and PSBS1 at the indicated conditions normalized to WT LL ( $n=3$ biological samples, mean $\pm s d)$. The $p$-values for the comparisons between the mutants and the WT are based on ANOVA Punnet's multiple comparisons test on $\log 10$ - transformed values and are indicated in the graphs $(* *, P<0.01)$.

88

89

90 
a

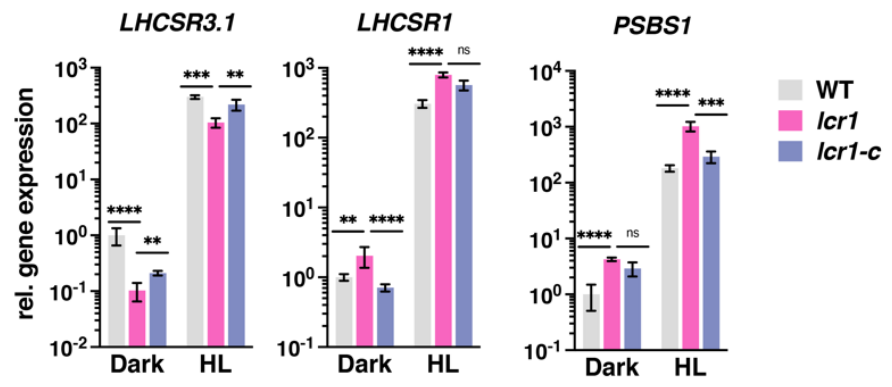

b

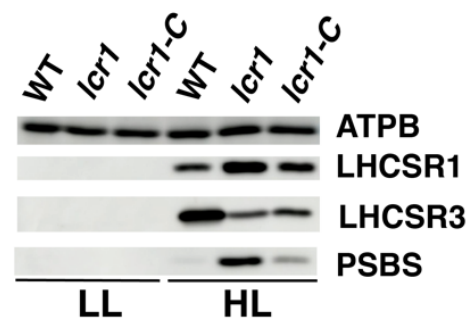

91
C

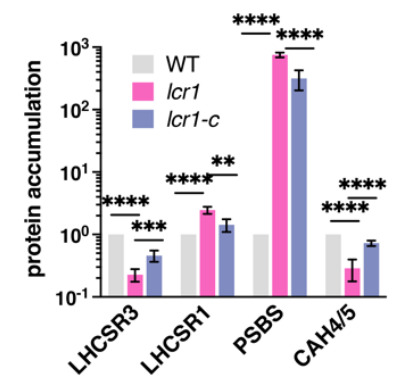

d

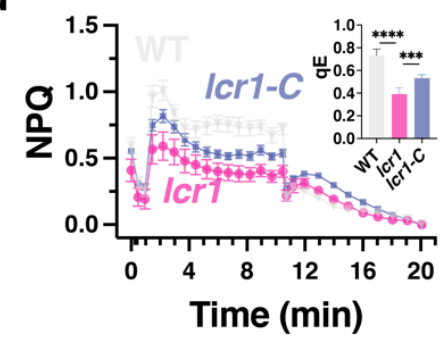

Extended Data Fig. 5: LCR1 is required for proper expression of qE-related genes during transitions from dark-to-light. WT, Icr1 and Icr1-C cells were acclimated for $16 \mathrm{~h}$ in darkness. After sampling for the dark conditions, light intensity was increased to $300 \mu \mathrm{mol}$ photons $\mathrm{m}^{-2} \mathrm{~s}^{-1}(\mathrm{HL})$; samples were taken $1 \mathrm{~h}$ (RNA) or 4 $\mathrm{h}$ (protein and photosynthetic measurements) after exposure to $\mathrm{HL}$. a. Relative expression levels of $\mathrm{qE}$ genes at the indicated conditions normalized to WT LL ( $n=3$ biological samples, mean $\pm s d)$. b. Immunoblot analyses of LHCSR1, LHCSR3, PSBS and ATPB (loading control) of one out of the three biological replicate samples, under the indicated conditions. c. Summary graph of immunoblots of all replicate samples of Extended Data Fig. $3 \mathrm{~b}$ after normalization to ATPB. Shown are the HL treated samples; WT protein levels were set as 1 . $\mathbf{d}$. NPQ and calculated qE $4 \mathrm{~h}$ after exposure to $\mathrm{HL}(n=3$ biological samples, mean $\pm s$.d). The $p$-values for the comparisons are based on ANOVA Dunnett's multiple comparisons test and are indicated in the graphs $(*, \mathrm{P}$ $<0.005, * *, \mathrm{P}<0.01, * * *, \mathrm{P}<0.001, * * * *, \mathrm{P}<0.0001)$. Statistical analyses for panel a and $\mathrm{c}$ were applied on $\log 10$ - transformed values. 
a

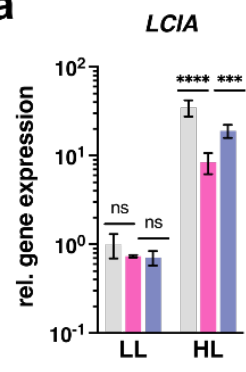

b

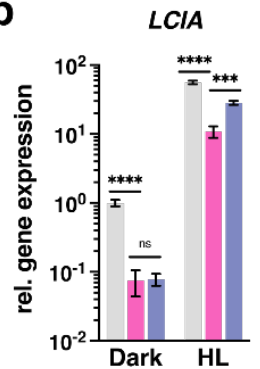

CAH4

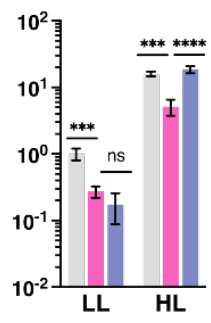

CAH4

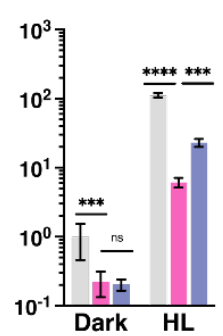

CCP1

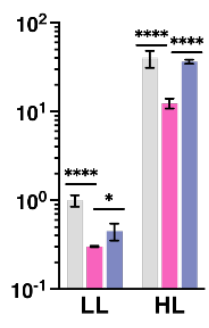

CCP1

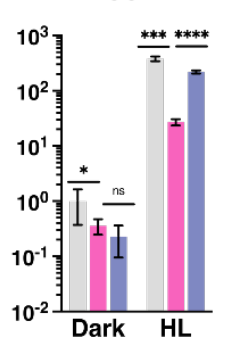

HLA3

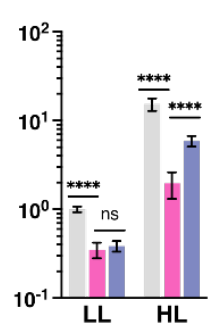

HLA3

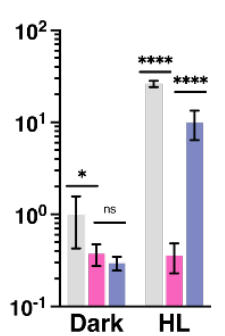

LCI1

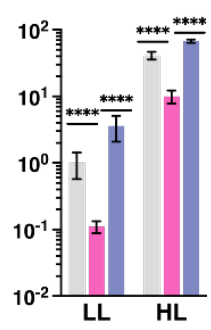

$L C I 1$

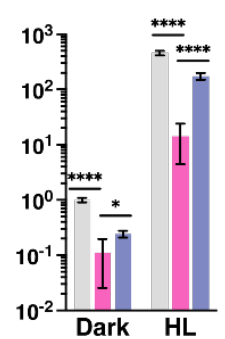

BST1

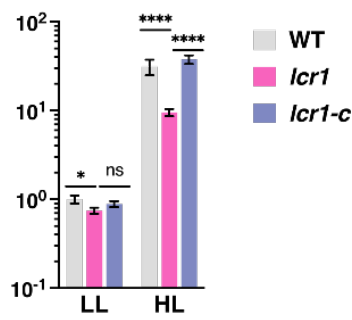

BST1

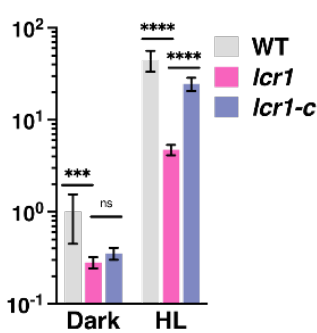

Extended Data Fig. 6: LCR1 activates the transcription of CCM genes in LL and dark-acclimated cells. a. WT, Icr1 and Icr1-C cells were acclimated for $16 \mathrm{~h}$ in $\mathrm{LL}\left(15 \mu \mathrm{mol}\right.$ photons $\left.\mathrm{m}^{-2} \mathrm{~s}^{-1}\right)$. After sampling under LL conditions, light intensity was increased to $300 \mu \mathrm{mol}$ photons $\mathrm{m}^{-2} \mathrm{~s}^{-1}(\mathrm{HL})$ and samples were taken $1 \mathrm{~h}$ after exposure to HL. Relative expression of CCM genes at the indicated conditions normalized to WT under LL. $\mathbf{b}$. Relative expression of CCM genes acclimated in $16 \mathrm{~h}$ of darkness ( $n=3$ biological samples, mean $\pm s d$ ). The $p$-values for the comparisons are based on ANOVA Dunnett's multiple comparisons test on log10- transformed values and are indicated in the graphs $(*, \mathrm{P}<0.005, * *, \mathrm{P}<0.01, * * *, \mathrm{P}<0.001, * * *, \mathrm{P}<0.0001)$. 
Extended Data Fig.7: Complementation of qer1 with QER1 (qer1-C strain) fails to rescue the LHCSR1 and PSBS phenotypes. WT, qer1 and qer1-C cells were acclimated for $16 \mathrm{~h}$ in $\mathrm{LL}\left(15 \mu \mathrm{mol}\right.$ photons $\left.\mathrm{m}^{-2} \mathrm{~s}^{-1}\right)$. After sampling for the dark conditions, light intensity was increased to $300 \mu \mathrm{mol}$ photons $\mathrm{m}^{-2} \mathrm{~s}^{-1}$ (HL); RNA samples were taken $1 \mathrm{~h}$ after exposure to $\mathrm{HL}$. Shown are relative expression levels of $\mathrm{qE}$ genes at the indicated conditions normalized to WT LL ( $n=3$ biological samples, mean $\pm \mathrm{sd}$ ). The $p$-values for the comparisons are based on ANOVA Dunnett's multiple comparisons test on log10-transformed values and are indicated in the graphs $(* * *, \mathrm{P}<0.001, * * * *, \mathrm{P}<0.0001)$. 
a

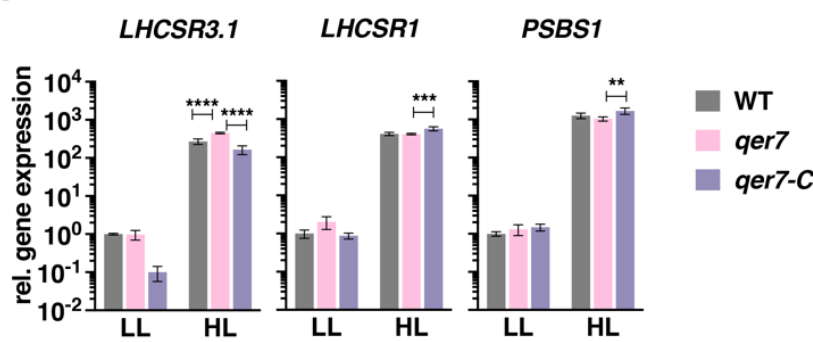

d

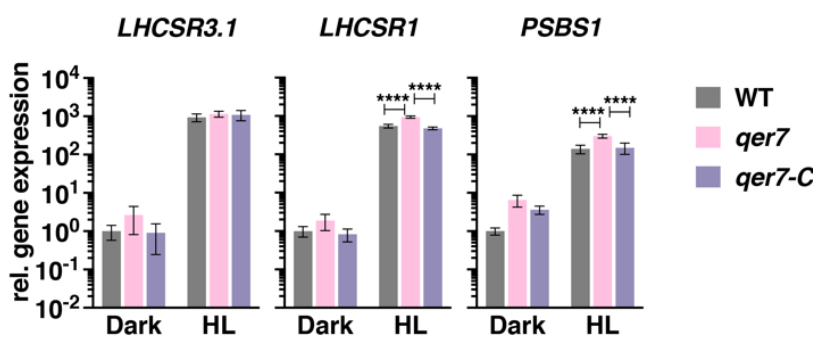

b

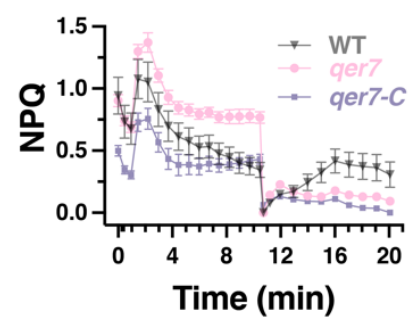

e

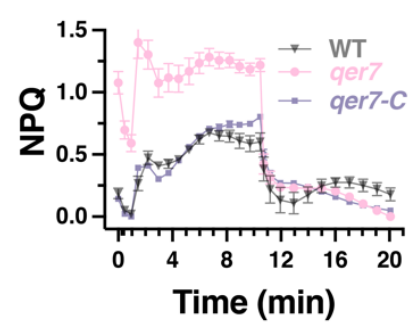

C

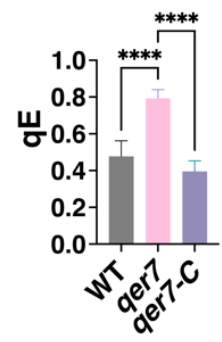

f

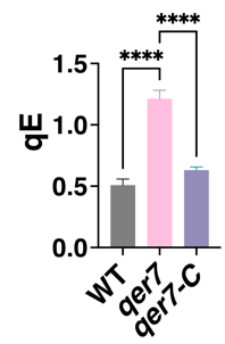

Extended Data Fig.8: Relative expression of qE-related genes in asynchronous qer7 cells. WT, qer7 and qer7$C$ cells were acclimated for $16 \mathrm{~h}$ in $\mathrm{LL}\left(15 \mu \mathrm{mol}\right.$ photons $\mathrm{m}^{-2} \mathrm{~s}^{-1}$; a-c) or darkness (d-f). After sampling for the LL or dark conditions, light intensity was increased to $300 \mu \mathrm{mol}$ photons $\mathrm{m}^{-2} \mathrm{~s}^{-1}(\mathrm{HL})$ and samples were taken $1 \mathrm{~h}$ (RNA) or $4 \mathrm{~h}$ (photosynthetic measurements) after exposure to $\mathrm{HL}$. a, d. Relative expression of qE and CCM genes at the indicated conditions normalized to WT LL (a) or dark (d) respectively ( $n=3$ biological samples, mean $\pm \mathrm{sd}$ ). b, e. NPQ and c, f. calculated $\mathrm{qE}$, $4 \mathrm{~h}$ after exposure to HL ( $n=3$ biological samples, mean \pm s.d. The $p$-values for the comparisons are based on ANOVA Dunnett's multiple comparisons test and are indicated in the graphs $(* *, \mathrm{P}<0.01, * * *, \mathrm{P}<0.001, * * * *, \mathrm{P}<0.0001)$. Statistical analyses for panel $\mathbf{a}$ and $\mathbf{d}$ were applied on $\log _{10^{-}}$transformed values. 
a

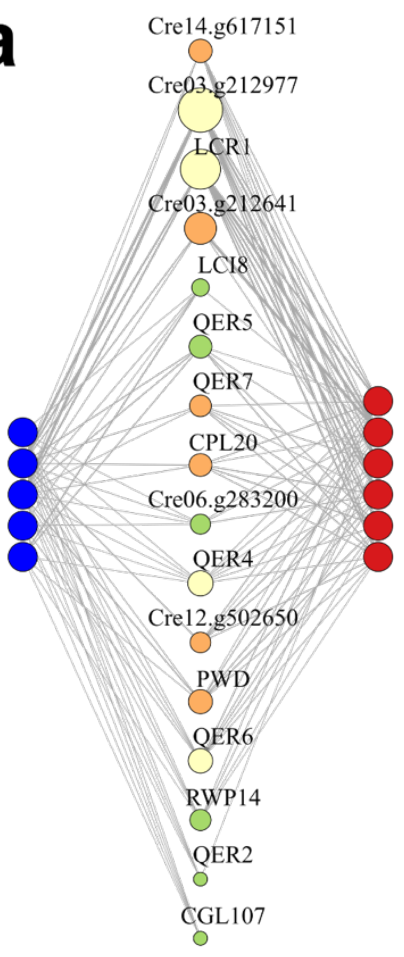

b

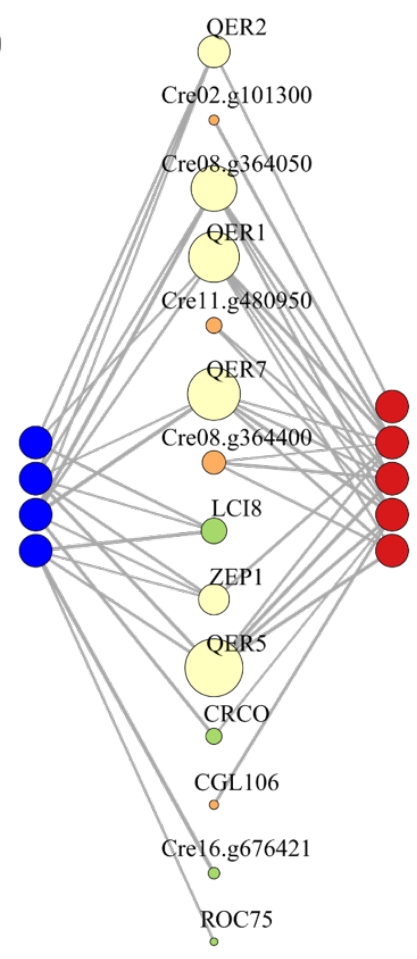

Extended Data Fig. 9: Reconstructed GRNs recapitulate experimentally found regulators of qE and CCM. Network representation of the top 10 regulators of CCM genes quantified via qPCR (Fig. 4), and qE genes as predicted from $\mathbf{a}$. the concensus or $\mathbf{b}$. PHOT-specific GRN. Regulators are represented by centeral nodes (same color code as in Fig. 5), target qE genes present in the respective network are shown in blue (LHCSR1, LHCSR3.1, LHCSR3.2, PSBS1, PSBS2) and target CCM genes are shown in red (LCI1, LCIA, CAH4, CCP1, HLA3, 
a

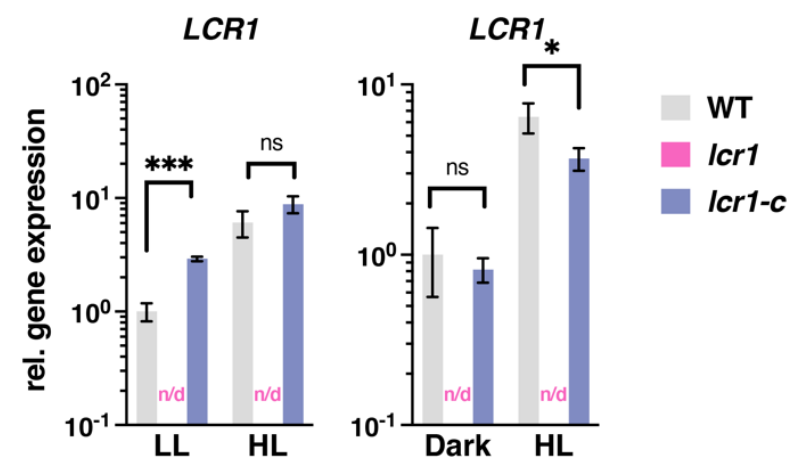

b

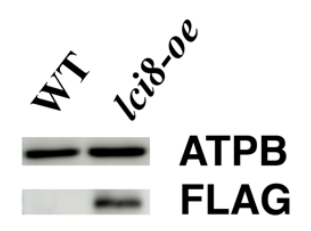

Extended Data Fig. 10: LCR1 mutation and LCI8 overexpression confirmation. a. Relative expression of LCR1 in WT, Icr1 and Icr1-C strains. Cells were acclimated for $16 \mathrm{~h}$ in LL or darkness. After sampling for the LL or dark conditions, light intensity was increased to $300 \mu \mathrm{mol}$ photons $\mathrm{m}^{-2} \mathrm{~s}^{-1}(\mathrm{HL})$, and samples for RNA purification were taken $1 \mathrm{~h}$ after exposure to HL ( $n=3$ biological samples, mean $\pm s d$ ). LCR1 gene expression was nondetectable (indicated as $\mathrm{n} / \mathrm{d}$ in the graphs) in the Icr1 mutant. b. Immunoblot analysis of LCI8-FLAG fused protein in WT and Ici8-oe cells grown in TAP, LL. The p-values for the comparisons are based on unpaired ttests on $\log 10$ - transformed values and are indicated in the graphs $(*, \mathrm{P}<0.005, * * *, \mathrm{P}<0.001)$. 


\section{Supplementary Table Captions}

172 Supplementary Table 1: RNAseq data sets used in this study; HSM: Sueoka's high salt medium (1); HSM new trace $=$ modified Sueoka's high salt medium with trace elements as described in (2); Rep= biological replicates

(1) Sueoka, N. Mitotic replication of deoxyribonucleic acid in Chlamydomonas reinhardii. Proc. Natl. Acad. Sci. U.S.A. 46, 83-91 (1960).

(2) Kropat, J.et al. A Revised Mineral Nutrient Supplement Increases Biomass and Growth Rate in Chlamydomonas Reinhardtii. Plant J. 2011, 66 (5), 770.

Supplementary Table 2: List of Transcription factors used in this study; Family info was adapted from PérezRodríguez et al. (2010) and Jin et al. (2017)

Supplementary Table 3: Edge list representation of the consensus network with mean ranks resulting from Borda count election method (Borda (1781), Marbach et al. (2011)) as edge attributes.

Supplementary Table 4: Top 10 predicted regulators of qE genes in the consensus GRN. Regulation strength was calculatedd based on the edge rank in the consensus network.

Supplementary Table 5: Edge list representation of the PHOT GRN with importance score from GENIE 3 (Huyn-Thu et al., 2010) as edge attributes.

Supplementary Table 6: Top 10 predicted regulators of qE genes in the PHOT GRN. Regulation strength was calculated based on the edge weight assigned by GENIE3.

Supplementary Table 7: List of genes putatively involved in photoprotection used for regulator prediction

Supplementary Table 8: List of genes putatively involved in CCM used for regulator prediction.

Supplementary Table 9: PCR primers for CLiP mutant validation and complementation in this study 